\title{
Influential Article Review - Did Bosses Prefer Applications From Non-Immigrant Families? An Interconnected Firm-Applicant Survey Is Used to Look into Access to Skills Courses Among Poor Youth
}

\author{
Giuseppe Beech \\ Kareem Cooley \\ Kylie Buckner
}

This paper examines apprenticeship and recruiting preferences. We present insights from a highly influential paper. Here are the highlights from this paper: In the German system of dual vocational training, in which companies recruit apprentices, graduates from low-level secondary schools (Hauptschule), and particularly those from immigrant families, are at a significant disadvantage regarding access to such apprenticeships. Previous qualitative studies have already pointed to the role of companies' recruiting criteria for mechanisms of indirect discrimination, e.g., their desire for smooth social interaction within the firm. This article builds on a standardized survey among companies to which low-skilled adolescents successfully or unsuccessfully applied for dual vocational training. The company data are matched with secondary longitudinal survey data on the same girls and boys. Which recruiting criteria put ethnic minorities at a disadvantage and hence indirectly facilitate ethnic discrimination? The theoretical approach considers (knowledge of) the immigration country's official language as both a functional requirement and a tool of hierarchical ethnic boundary making. In addition, concepts of social interaction within firms and respective norms as well as of homophily are subjected to an empirical test. Statistical results show that the below-average recruitment chances of applicants with a migration background are somewhat lower if companies stress a social fit criterion in their selection procedures. Furthermore, contrary to the initial hypothesis, ethnic minority youths are only substantially disadvantaged when applying to firms which consider the ability of verbal expression less crucial. Firms which consider this criterion very important may give applicants from immigrant families the chance to present themselves in a job interview and thus dispel ethnic stereotypes like the one that second-generation immigrant youths display serious language deficiencies. For our overseas readers, we then present the insights from this paper in Spanish, French, Portuguese, and German.

Keywords: dual vocational training, apprenticeships, ethnic minority, second generation immigrant, lowlevel school education, recruiting preferences, recruiting decisions, company survey 


\section{SUMMARY}

- Before turning to the hypotheses on indirect mechanisms of ethnic discrimination, let us consider the possibility of measuring ethnic stereotypes and more specifically their potential direct effect on recruiting decisions. One section of the company survey asks the interviewees to state how far they agree with general statements which do not refer to themselves or their company in a straightforward way. Depending on the specific statements, differing shares of interviewed firms agreed that adolescents from immigrant families have «trouble» with the German language, cause problems in interaction with clients, are negative for social cohesion among apprentices, or that they attract clients of the same ethnic origin. A majority disagreed with the statement that most clients have no problem when a girl wears a headscarf. But there are no significant relationships between these five items und recruitment results; further, there is U-shaped relationship between the former and an index of social desirability. In terms of the full ethnic penalty, one should think of these two results as belonging together.

- How are these disparities to be explained? It is not the case that the compared groups of companies differ in their general opinion about ethnic minority adolescents' deficiencies in German, given their answers to the respective question mentioned above. A probable causal mechanism could be that firms which regard this linguistic capacity as very important had a closer look at the style and immaculateness of the written applications and invited applicants of native and immigrant origin who fulfilled their minimum standard. Subsequently, when ethnic minority applicants had the opportunity to present themselves in an interview and the HR personal could confirm that their verbal expression was proficient, the applicants had the same chance to become an apprentice as their native peers. Hence, these companies might have actually evaluated this language-specific criterion, i.e., the functional side of language, in a meritocratic way. The other firms might have just assumed linguistic deficiencies without evaluating them rigorously.

- The two company-level factors studied here correlate only slightly. The businesses for which the ability of expression was a very important selection criterion had above-average shares of immigrants in their workforces, which probably contributed to unbiased recruiting behavior. They belonged more often to the service sector - maybe because communication with clients plays a more important role here than for occupations within the industrial and craft sectors. Among companies that did not consider the social fit criterion to be very important, firms in the craftsthe large majority with smaller numbers of employees-were less common and those in the industrial sector more common; the share of immigrants among employees was more often above average than among companies which deemed social fit very important. These structural factors by themselves, however, did not exert a significant impact on the applicants' success in this particular sample.

\section{HIGHLY INFLUENTIAL ARTICLE}

We used the following article as a basis of our evaluation:

Söhn, J. (2020). Why companies prefer applicants from non-immigrant families: investigating access to vocational training among low-qualified adolescents with an interlinked firm-applicant survey. Empirical Research in Vocational Education and Training, 12(1).

This is the link to the publisher's website:

https://ervet-journal.springeropen.com/articles/10.1186/s40461-020-00090-Z 


\section{INTRODUCTION}

Germany is a prime example of a coordinated economy with a large segment of vocational educational training (VET) within the national education system. Companies are a major actor regarding the organization of apprenticeships in the dual VET system, which take place both on the job in a firm and in occupational schools. Young people striving for this qualification have to apply individually to companies that offer apprenticeships. It is the company - not vocational schools - that may choose among applicants. Hence, firms act as gatekeepers to such vocational training. This articles focuses on how such gatekeeping operates. The matching of company and trainee is subject to market mechanisms such as employee recruitment procedures. This logic of selection is characterized by the relation between supply and demand, by applicant queues with more or less favorable positions, and by the risk of discrimination based on ascriptive features like gender or ethnic background.

In legal terms, there are no preconditions regarding educational credentials - be it the kind of secondary school diploma or the average grade attained - with respect to most types of apprenticeships in Germany. In reality, however, this important transition in adolescents' life course from secondary school to occupational training very much depends on the kind of diploma that applicants graduate with (see, e.g., Autorengruppe Bildungsberichterstattung 2018; Baas and Philipps 2019; Protsch and Solga 2016; Erdmann 2016). Within the highly stratified secondary school system in Germany, graduates with a low-level secondary school diploma (Hauptschulabschluss) face severely lower chances of starting an apprenticeship than those with a mid-level school diploma (Realschulabschluss/Mittlere Reife) or a high-level school diploma (Abitur), which also allows access to tertiary education. Post-school qualification, including its level and occupational specialization, strongly influences employment opportunities, job quality, and financial gratification in their subsequent life course.

The kind of secondary-school diploma that applicants for dual VET can present to potential employers serves the latter as a signal for the applicant's human capital and learning capacity-to some extent a justified signal. Unequal treatment based on ascriptive characteristics, in contrast, is neither legitimate nor legal. In this regard, adolescents from immigrant families or-depending on the empirical operationalization - of non-native citizenship are disadvantaged in gaining access to VET in Germany (Autorengruppe Bildungsberichterstattung 2018; Beicht and Walden 2018, 2019; Hunkler 2016; BMBF 2019; see also various chapters in the edited volume by Scherr 2015). Similar ethnic disparities have been found in other countries with a strong dual VET system like Switzerland (Seibert et al. 2009). In addition to high bivariate correlations, these studies show that applicants from ethnic minorities face a higher risk of remaining without VET and having to resort to preparatory prevocational programs even when previous academic performance is statistically controlled for, i.e., when the studies account for the fact that ethnic minority students already have less opportunity to reach higher-level diplomas and better grades than peers of native parentage. Among youths with a migration background no longer attending school, $40 \%$ of 15 - to 19 -year-olds and $28 \%$ of 20 - to 24 -year-olds have at most a low-level secondary school diploma. These are substantially larger shares than among native peers (24 and 15\%, respectively; see Autorengruppe Bildungsberichterstattung: Tab. B5-5web, author's calculation).

This article focuses on applicants to dual VET who are multiply disadvantaged in terms of low-level secondary schools and ethnic minority status, and empirically investigates the firm-applicant linkage with regard to recruiting practices and results. As will be elaborated in the "Previous insights into ethnic and education-related disadvantages in VET" section, research has until now investigated ethnic disparities in VET on the side of either applicants or companies. This study uses a unique database that merges a survey on companies with one on applicants who graduated from low-level schools in the German regional state of Lower Saxony and applied successfully or unsuccessfully to the aforementioned companies.

In order to detect discrimination, several studies have used correspondence tests with fake applications [regarding apprenticeship in Germany, see Protsch (2017) with respect to organizational features and Protsch and Solga (2017) regarding discrimination based on immigrant status; regarding college students' internships in Germany, see Kaas and Manger (2012); on ethnic discrimination in the labor market, see Thijssen et al. (2019) for Germany compared to the Netherlands and Zschirnt (2019) for Switzerland]. In 
comparison to such field experiments, the data used here reflect a greater measure of external validity because they capture the real action of companies in terms of actual recruitment. Thus, this study can better answer the following research questions: to what extent do companies' recruiting preferences influence which types of graduates from low-level secondary schools they select for apprenticeship, and which graduates remain without vocational training? Which company preferences interact with the high risk of adolescents from immigrant families remaining without an apprenticeship?

Given that outright racist justifications for not recruiting ethnic minorities are illegal and (for many) socially unacceptable, indirect and less conscious forms of unequal treatment appear more likely. Qualitative studies using in-depth interviews with actors in companies responsible for hiring apprentices show how some of them voice concealed forms of ethnic prejudice. For instance, they refer to bad experiences with "foreign" apprentices even though it turns out later in the interview that they have never actually employed one but merely "heard" about trouble (Imdorf 2007; see also Schneider et al. 2015: 418 with regard to Turkish-origin applicants to German companies). As "Conceptual approaches to companies as gatekeepers in dual VET" section will argue, companies' preference for familiarity and homogeneity within their workforce as well as good German language skills - both a functional requirement and an ethno-national marker - may act as indirect avenues leading to the overrepresentation of apprentices from native families. "Methods" section presents the database and operationalization. "Results and discussion" section reports the extent to which firms share stereotypical views on migrants and then tests two hypotheses empirically. "Conclusions" section summarizes the main findings and gives an outlook on future research and policy options.

\section{CONCLUSION}

This article has used a unique set of interlinked firm-applicant data in order to investigate crucial mechanisms of indirect ethnic discrimination in dual VET in Germany. The analysis yielded evidence of how ethnic bias in recruiting behavior works through the connection between companies' recruiting preferences and the ascribed features of ethnic minority applicants. Meanwhile, multivariate models held important educational signals like previous participation in prevocational programs constant.

Previous qualitative findings (Imdorf 2007, 2010) suggest that firms prefer harmony and familiarity in the domestic world of the company. In order to avoid creating conflict or prematurely ending an apprenticeship, thus spoiling their investment in the "wrong" applicants, companies are believed to shy away from those of foreign parentage as a "preventative measure". The quantitative firm-applicant survey, however, found only some evidence that a firm's relative emphasis on how well applicants "fit into the team" might have an influence on ethnic minority adolescents' chances of being recruited. Future research with a higher number of cases and better possibilities to differentiate should test whether the assumed effect is particularly relevant for ethnic minority youths with a high risk of ethno-racial stigmatization, such as those identifiable by a "non-German" name of Turkish or Arab origin, darker skin color, or a head scarf.

Second, companies which highly value the selection criterion of good verbal expression may organize their recruitment process in a way that gives applicants from immigrant families the opportunity to prove, e.g., in job interviews, that their German is proficient, counter to prejudiced assumptions about immigrants and second-generation youths. As to the companies for which linguistic abilities are somewhat less important, it seems plausible that prejudice regarding ethnic minority graduates' linguistic skills feeds into a larger skepticism about them, which in turn translates into below-average training rates. When asked about problems with "weaker" apprentices, only 5\% of German firms who had had ethnic minority apprentices named language-related obstacles in the survey of Enggruber and Rützel (2015: 41). Furthermore, in the survey analyzed here, only $2 \%$ of companies that had previously trained apprentices with a migration background would not do this again.

In terms of methodological limitations, the major drawbacks of the database used here are the - for quantitative analysis - small number of cases as well as the lack of formal representativeness. Hence, it remains unclear in a number of instances whether the lack of statistically significant correlations between potentially influential factors and the ethnic minority-majority gap is only due to the sample size or to a 
systematic similarity between groups. Still, interlinking the panel survey on applicants with the company survey is a very suitable design for tackling research questions like those of this article. The external validity is clearly higher than experimental studies which can follow the success of "fake" applications only to the point of an invitation to a job interview but not to the applicants' actual employment, as this study could. However, in terms of methodological lessons for the future, it seems advisable to add such a company survey to an already existing representative large-scale study with a special focus on school-to-VET-andwork transitions such as the German National Educational Panel Study (Starting Cohort 9th Grade). 15 Furthermore, future research may try to measure the quality of written applications (in terms of, e.g., typos and adequate language style) and its effect on invitations to job interviews and on actual recruitment, as many schools and support programs teach students how to write "good" applications.

The analysis revealed that many recruiting preferences measured in the survey show little variance between firms. This renders them statistically unsuitable as predictors even though very common selection criteria like the candidate's reliability and motivation (Krug von Nidda 2019: 27) - and especially their perception by the company-surely do play an important part in the recruiting process. Apart from structural characteristics of the company (e.g., the apprenticeship supply-and-demand ratio and economic sector), information like the company's recruitment of candidates through traineeships or the selection criterion of the "general impression" given by written applications did not hold as significant predictors in multivariate estimation models. The fact that many criteria named as important for selecting apprentices do not discriminate between applicants from the ethnic majority and minorities is mirrored in another company survey: Enggruber and Rützel (2015: 37) also find no significant difference between firms which did or did not have apprentices from immigrant families during the preceding 5 years - neither with regard to selection criteria nor to suggestions for improving the quality and success rate of dual VET in general. Some items which were thought to potentially put ethnic minority applicants at a disadvantage - like "gut feeling" (author's translation from the questionnaire) - show no bivariate correlation to speak of. The wording of items is obviously important; it must be sensitive to social norms which discourage HR personnel from voicing opinions that sound outright non-meritocratic or unprofessional. These methodological problems in standardized surveys suggest that qualitative research, which can go deeper, is still needed in this field. Yet, as Imdorf (2010: 98) has suggested, the next step — however difficult to realize — should be participant observation in job interviews or in meetings when HR personnel discuss interviewed candidates.

Given that almost all companies which have already trained apprentices from immigrant families would not mind doing it again, those without such experience should be encouraged to give it a try. Demographic aging and the expansion of tertiary education will further decrease the number of applicants for VET. The shorter the line of applicants, the more willing some firms might become to "try out" an ethnic minority apprentice for the first time and hence break the ice for others later on. The results presented here suggest that inviting such candidates and listening to them might already contribute to diffusing potential reservations on the firm's part that oral German language skills could be deficient.

Furthermore, important actors in the political arena like the German Trade Union confederation and the Federal Anti-Discrimination Agency have been demanding for quite some time that at least large- and midsized companies should adopt anonymized application procedures (FAZ 2019)-otherwise still the exception in Germany. Such recruiting practices tend to improve equal opportunity for various at-risk groups, including individuals with a migration background (for the evaluation of such a pilot project among large German firms, see Krause et al. 2012: 9-10). Most ethnic minority applicants for VET have gone through the German school system from kindergarten onwards and would probably profit from anonymized application procedures, though immigrants' foreign educational career (like that of recent refugees) can hardly be disguised in a CV, pointing to the limitations of such a tool. Finally, regional states should invest more resources into the educational opportunities of immigrant children and the children of immigrants in primary and secondary education in order to boost their chances of accessing dual VET. 


\section{APPENDIX}

TABLE 1

SAMPLE STRUCTURE: DISTRIBUTION OF APPLICANTS ACROSS FIRMS AND VICE VERSA

\begin{tabular}{|l|l|l|l|l|}
\hline & \multicolumn{2}{|l|}{ Firms applied to per applicant } & \multicolumn{2}{l|}{ Applications per firm } \\
\cline { 2 - 5 } & n & \% (column) & n & \% (column) \\
\hline 1 & 454 & 76.2 & 503 & 84.4 \\
\hline 2 & 109 & 18.3 & 57 & 9.6 \\
\hline 3 to 4 & 33 & 5.5 & 18 & 3.0 \\
\hline 5 to 11 & - & - & 18 & 3.0 \\
\hline Total & 596 & 100.0 & 596 & 100.0 \\
\hline
\end{tabular}

FIGURE 1

ESTIMATING THE PROBABILITY OF SUCCESS AT INTERVIEWED FIRMS AND OF UNSUCCESSFUL SCHOOL-TO-VET TRANSITIONS: COMPANIES' OPINION ON THE SELECTION CRITERION “ABILITY TO EXPRESS ONESELF” INTERACTED WITH

APPLICANTS' ETHNIC BACKGROUND (AVERAGE MARGINAL EFFECTS IN COMPARISON TO ALL APPLICANTS OF NATIVE PARENTAGE)

Firm: criterion less important $\&$ at least one parent foreign-born/missing
Firm: criterion very important $\&$ at least one parent foreign-born/missing

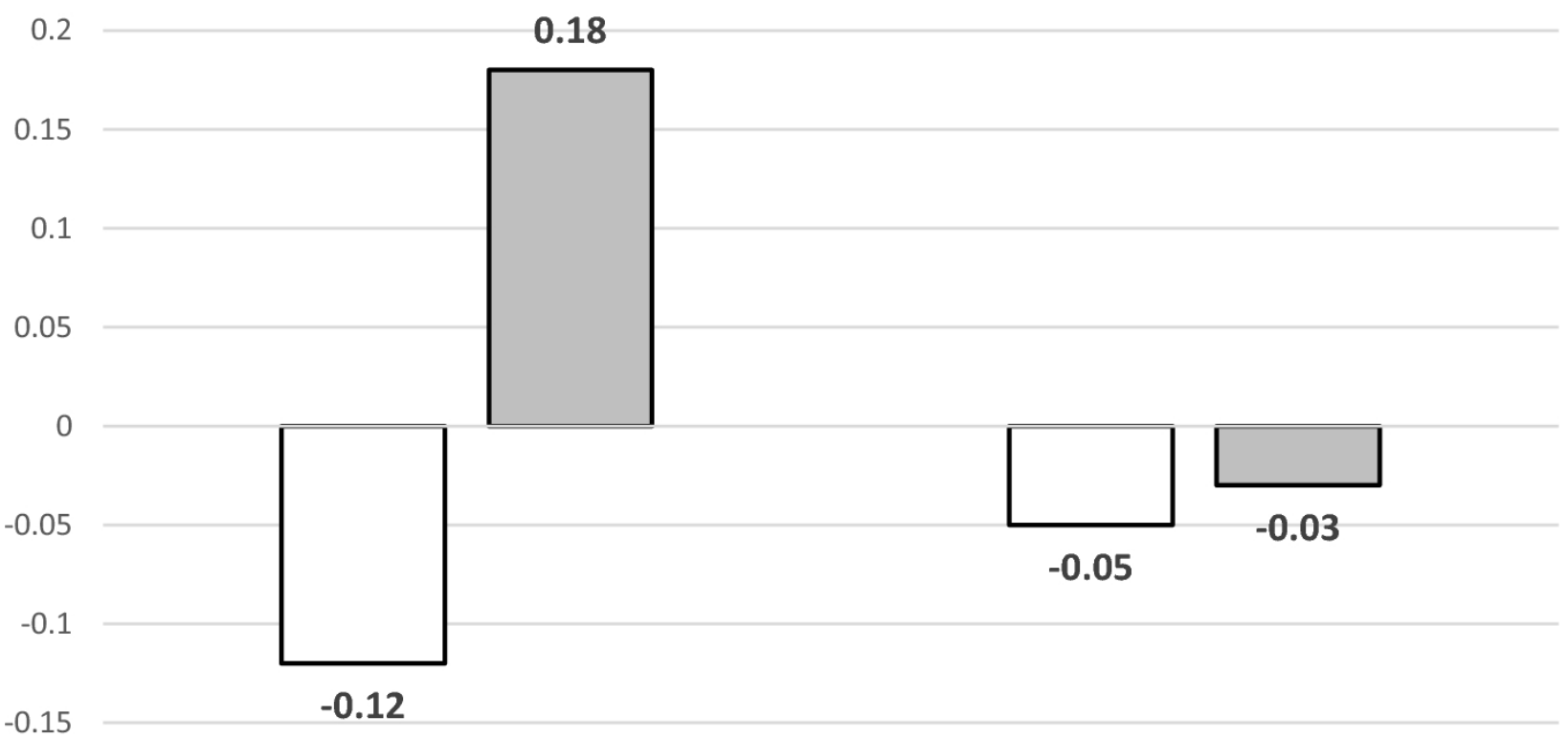

$\square$ successful at interviewed company unsuccessful school-to-VET transition

(Source: Own interlinked company-applicant survey; author's calculation)

$\mathrm{N}=596$ company-applicant dyad; see also Table 2 


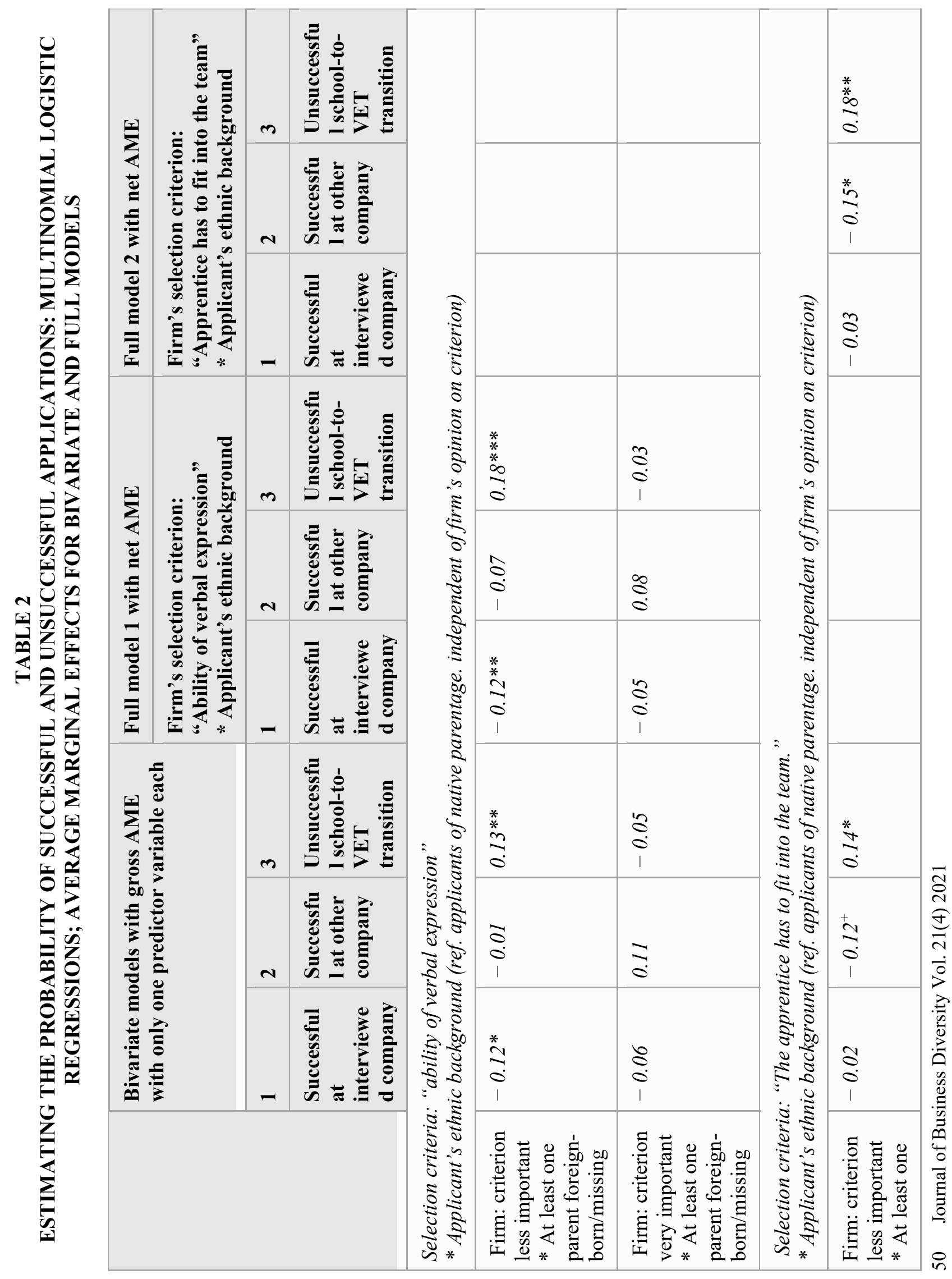




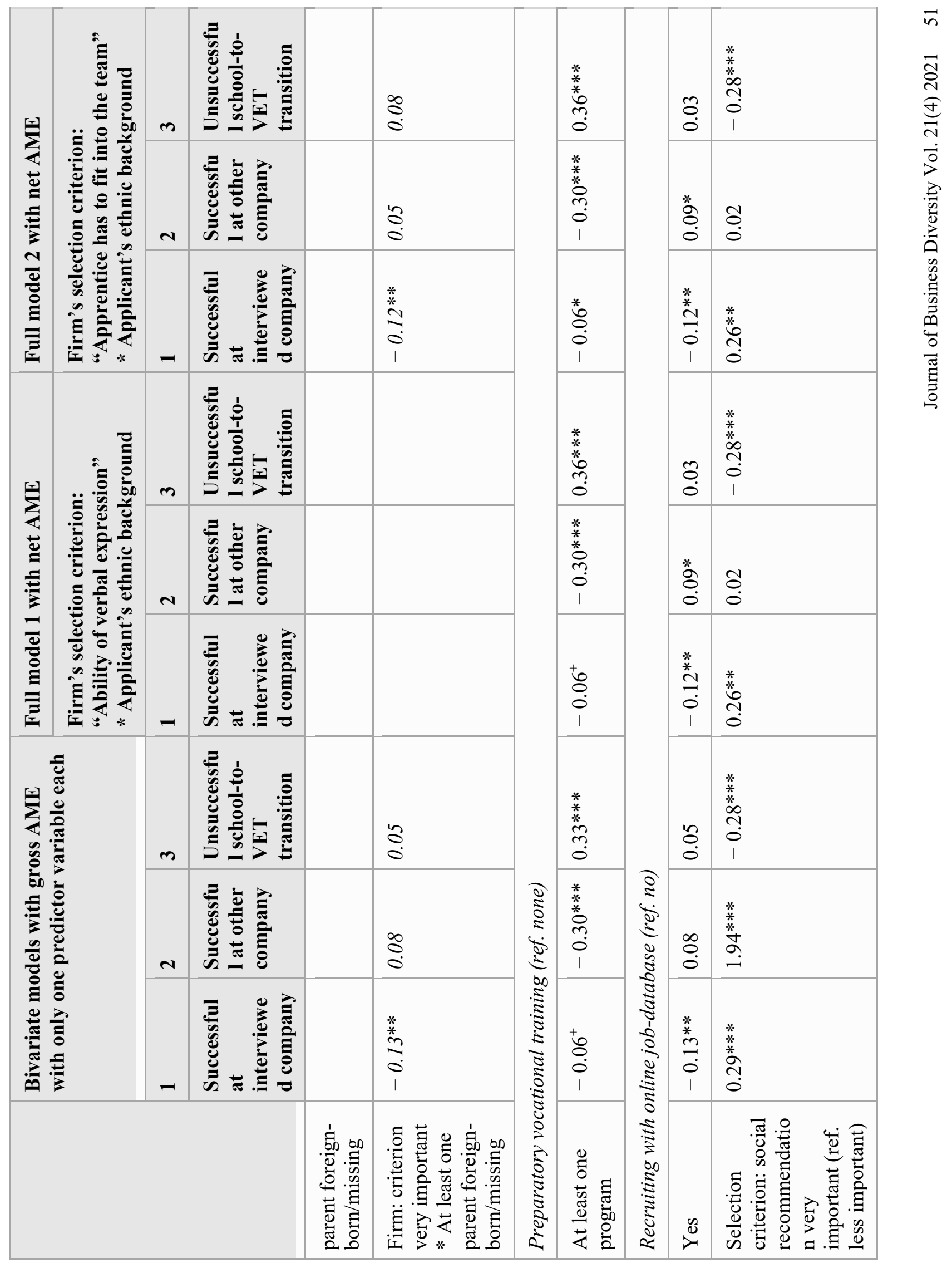




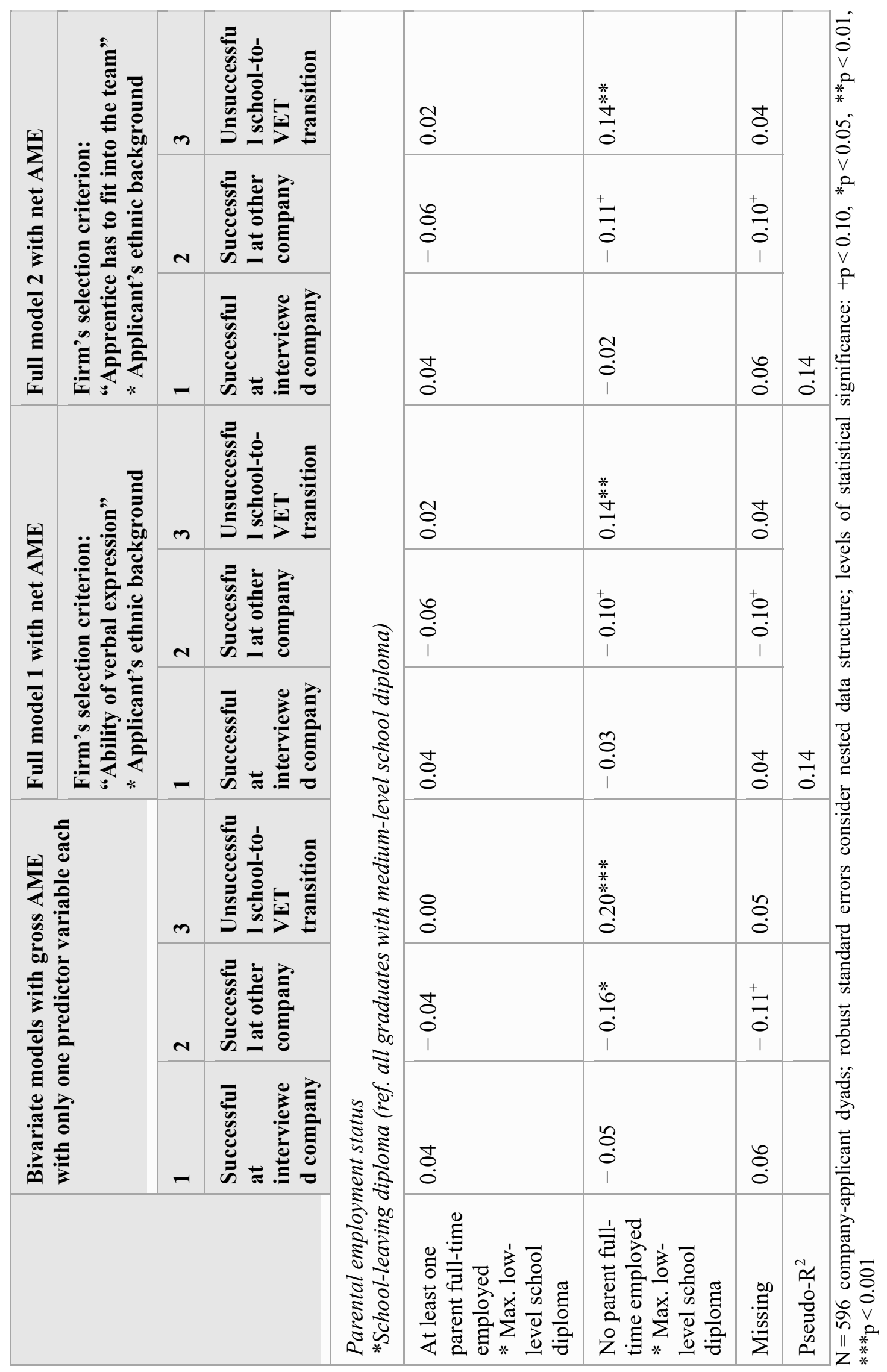

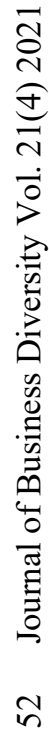




\section{FIGURE 2}

ESTIMATING THE PROBABILITY OF AT INTERVIEWED FIRM AND OF UNSUCCESSFUL SCHOOL-TO-VET TRANSITIONS: COMPANIES' OPINION ON THE SELECTION CRITERION “APPRENTICE FITS WELL INTO THE TEAM” INTERACTED WITH APPLICANTS' ETHNIC BACKGROUND (AVERAGE MARGINAL EFFECTS IN COMPARISON TO ALL APPLICANTS OF NATIVE PARENTAGE)

Firm: criterion less important \& at least one parent foreign-born/missing
Firm: criterion very important $\&$ at least one parent foreign-born/missing

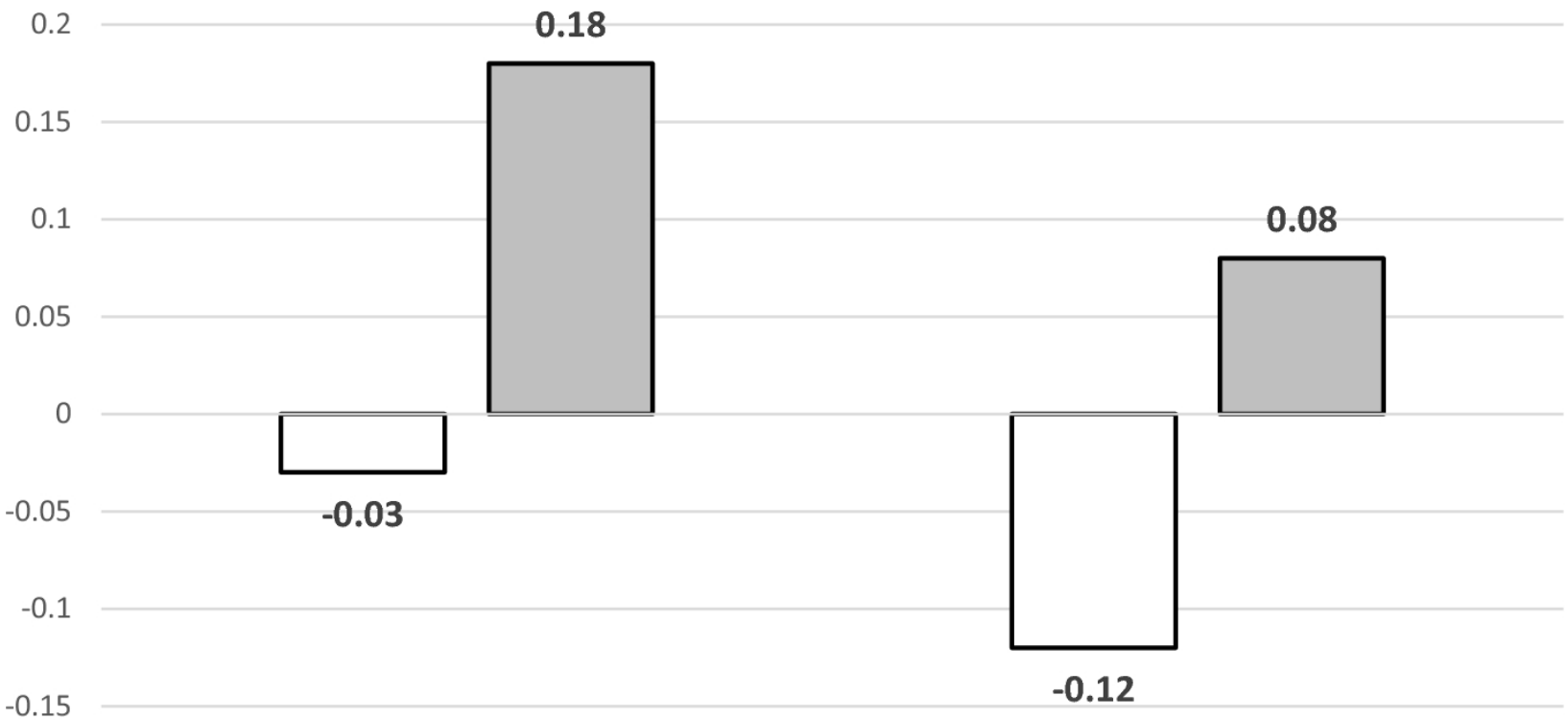

$\square$ successful at interviewed company $\quad \square$ unsuccessful school-to-VET transition

(Source: Own interlinked company-applicant survey; author's calculation)

$\mathrm{N}=596$ company-applicant dyads; see also Table 2 
TABLE 3

\section{ALTERNATIVE REFERENCE CATEGORIES WHEN ESTIMATING THE PROBABILITY OF SUCCESSFUL AND UNSUCCESSFUL APPLICATIONS: MULTINOMIAL LOGISTIC REGRESSIONS; AVERAGE MARGINAL EFFECTS FOR FULL MODELS}

\begin{tabular}{|c|c|c|c|c|c|c|}
\hline & \multicolumn{3}{|c|}{ Full model 1 with net AME } & \multicolumn{3}{|c|}{ Full model 2 with net AME } \\
\hline & \multicolumn{3}{|c|}{$\begin{array}{l}\text { Firm's selection criterion: } \\
\text { "Ability of verbal expression" } \\
\text { *Applicant's ethnic background }\end{array}$} & \multicolumn{3}{|c|}{$\begin{array}{l}\text { Firm's selection criterion: } \\
\text { "Apprentice has to fit into the team." } \\
\text { *Applicant's ethnic background }\end{array}$} \\
\hline & 1 & 2 & 3 & 1 & 2 & 3 \\
\hline & $\begin{array}{l}\text { Successful } \\
\text { at } \\
\text { interviewed } \\
\text { company }\end{array}$ & $\begin{array}{l}\text { Successful } \\
\text { at other } \\
\text { company }\end{array}$ & $\begin{array}{l}\text { Unsuccessful } \\
\text { school-to- } \\
\text { VET } \\
\text { transition }\end{array}$ & $\begin{array}{l}\text { Successful } \\
\text { at } \\
\text { interviewed } \\
\text { company }\end{array}$ & $\begin{array}{l}\text { Successful } \\
\text { at other } \\
\text { company }\end{array}$ & $\begin{array}{l}\text { Unsuccessful } \\
\text { school-to- } \\
\text { VET } \\
\text { transition }\end{array}$ \\
\hline \multicolumn{7}{|c|}{$\begin{array}{l}\text { Selection criteria: "ability of verbal expression" } \\
\text { * Applicant's ethnic background (ref. Firm: criterion less important * at least one parent foreign- } \\
\text { born/missing) }\end{array}$} \\
\hline $\begin{array}{l}\text { Applicants of } \\
\text { native parentage. } \\
\text { independent of } \\
\text { firm's opinion } \\
\text { on criterion }\end{array}$ & $0.11 * *$ & 0.06 & $-0.17^{* * *}$ & & & \\
\hline $\begin{array}{l}\text { Firm: criterion } \\
\text { very important } \\
\text { * At least one } \\
\text { parent foreign- } \\
\text { born/missing }\end{array}$ & 0.06 & 0.10 & $-0.16^{* *}$ & & & \\
\hline \multicolumn{7}{|c|}{$\begin{array}{l}\text { Selection criteria: "The apprentice has to fit into the team." } \\
\text { * Applicant's ethnic background (ref. Firm: criterion less important * at least one parent foreign- } \\
\text { born/missing) }\end{array}$} \\
\hline $\begin{array}{l}\text { Applicants of } \\
\text { native parentage. } \\
\text { Independent of } \\
\text { firm's opinion } \\
\text { on criterion }\end{array}$ & & & & 0.03 & $0.14 *$ & $-0.17 * *$ \\
\hline $\begin{array}{l}\text { Firm: criterion } \\
\text { very important } \\
\text { * At least one } \\
\text { parent foreign- } \\
\text { born/missing }\end{array}$ & & & & $-0.12^{*}$ & $0.20 * *$ & -0.08 \\
\hline
\end{tabular}

$\mathrm{N}=596$ company-applicant dyads; control variables are the same as in the full models in Table 2; robust standard errors consider nested data structure; levels of statistical significance: $+\mathrm{p}<0.10,{ }^{*} \mathrm{p}<0.05,{ }^{* *} \mathrm{p}<0.01$, $* * * \mathrm{p}<0.001$ 


\section{REFERENCES}

Arrow, K.J. (1998). What has economics to say about racial discrimination? J Econ Perspect, 12(2), 91100.

Autorengruppe Bildungsberichterstattung. (2008). Bildung in Deutschland 2008. Ein indikatorengestützter Bericht mit einer Analyse zu Übergängen im Anschluss an den Sekundarbereich I. Bertelsmann, Bielefeld.

Autorengruppe Bildungsberichterstattung. (2016). Bildung in Deutschland 2016. Ein indikatorengestützter Bericht mit einer Analyse zu Bildung und Migration. Bertelsmann, Bielefeld.

Autorengruppe Bildungsberichterstattung. (2018). Bildung in Deutschland 2018. Ein indikatorengestützter Bericht mit einer Analyse zu Wirkungen und Erträgen von Bildung. Bertelsmann, Bielefeld.

Baas, M., \& Philipps, V. (2019). Über Ausbildung in Arbeit? Verläufe gering gebildeter Jugendlicher. In Forschungsverbund Sozioökonomische Berichterstattung (Ed.), Berichterstattung zur sozioökonomischen Entwicklung in Deutschland (pp. 411-446). Exklusive Teilhabe - ungenutzte Chancen. Dritter Bericht, Bertelsmann, Gütersloh.

Beicht, U., \& Walden G. (2018). Übergänge nicht studienberechtigter Schulabgänger/-innen mit Migrationshintergrund in vollqualifizierende Ausbildung. BIBB-Report 6|2018, Bundesinstitut für Berufsbildung (BIBB), Bonn.

Beicht, U., \& Walden, G. (2019). Der Einfluss von Migrationshintergrund, sozialer Herkunft und Geschlecht auf den Übergang nicht studienberechtigter Schulabgänger/-innen in berufliche Ausbildung. BIBB Wissenschaftliche Diskussionspapiere Heft 198, BBIB, Bonn.

Berry, W.D., DeMeritt, J.H.R., \& Esarey, J. (2010). Testing for interaction in binary logit and probit models: is a product term essential? Am J Polit Sci, 54(1), 48-266.

BMBF (Bundesministerium für Bildung und Forschung). (2019). Berufsbildungsbericht 2019. Berlin.

Boltanski, L., \& Thevenot, L. (2006). On justification: Economies of worth. Princeton University Press, Princeton.

Buck, E. (2011). Asymmetrische Gemeinschaft. "Integration” als leerer Signifikant im Zugehörigkeitsdiskurs Deutschlands und Großbritanniens. Sociol Int, 49(1), $29-52$.

Chullen, C.L., Adeyemi-Bello, T., \& Vermeulen, E. (2017). A Comparative Analysis of Attitudes Towards Women as Managers in the U.S. and Netherlands. Journal of Leadership, Accountability and Ethics, 14(2). Retrieved from https://articlegateway.com/index.php/JLAE/article/view/1601

Diehl, C., Friedrich, M., \& Hall, A. (2009). Jugendliche ausländischer Herkunft beim Übergang in die Berufsausbildung: Vom Wollen, Können und Dürfen. Zeitschrift für Soziologie, 38(1), 48-67.

Enggruber, R., \& Rützel, J. (2015). Berufsausbildung junger Menschen mit Migrationshintergrund. Eine repräsentative Befragung von Betrieben. Bertelsmann Stiftung, Gütersloh.

Erdmann, N. (2016). Erfolgreiche Wege bildungsbenachteiligter Jugendlicher in dualen Ausbildungen Rekonstruktionen zu Erfolgsbedingungen aus biographieanalytischer Perspektive. In B. van Dausien, et al (Eds.), Bildungswege. Biographien zwischen Teilhabe und Ausgrenzung (pp. 333361). Frankfurt a.M., Campus.

Esser, H. (2006). Sprache und Integration. Die sozialen Bedingungen und Folgen des Spracherwerbs von Migranten. Campus, Frankfurt.

Frankfurter Allgemeine Zeitung (FAZ). (2019). Unternehmen haben bei anonymen Bewerbungen noch Luft nach oben. Retrieved February 5, 2020, from https://www.faz.net/einspruch/beruf-undausbildung/antidiskriminierungsstelle-unternehmen-haben-bei-anonymen-bewerbungen-luftnach-oben-16604321.html

Gerdes, J., \& Bittlingmayer, U.H. (2011). Assimilations und Wissensgesellschaft. Bildungsgesteuerte Integrationsimperative im deutschen parteipolitischen Diskurs seit den Debatten um das Zuwanderungsgesetz. Sociol Int, 49(1), 03-138. 
Hunkler, C. (2016). Ethnische Unterschiede beim Zugang zu beruflicher Ausbildung. In C. Diehl, et al. (Eds.), Ethnische Ungleichheiten im Bildungsverlauf (pp. 597-641). Mechanismen, Befunde, Debatten. Springer, Wiesbaden.

Imdorf, C. (2007). Individuelle oder organisationale Ressourcen als Determinanten des Bildungserfolgs? Organisatorischer Problemlösungsbedarf als Motor sozialer Ungleichheit. Swiss J Sociol, 33(3), 407-423.

Imdorf, C. (2010). Emotions in the hiring procedure: How 'gut feelings' rationalize personnel selection decisions. In B. Sieben \& Ä. Wettergren (Eds.), Emotionalizing organizations and organizing emotions (pp. 94-105). Palgrave Macmillan, Houndmitls.

Kaas, L., \& Manger, C. (2012). Ethnic discrimination in Germany's labour market: A field experiment. German Econ Rev, 13(1), 1-20.

Kohlrausch, B. (2012). Betriebe als Gatekeeper. Sozialer Fortschritt, 61(10), 257-265.

Kohlrausch, B., \& Richter, M. (2016). Was fördert die nachhaltige Integration von Hauptschüler/linnen in den Ausbildungsmarkt? Arbeit. Zeitschrift für Arbeitsforschung, Arbeitsgestaltung und Arbeitspolitik, 25(3-4), 147-168

Krause, A., Rinne, U., \& Zimmermann, K.F. (2012). Anonymous job applications in Europe. IZA J Eur Labor Stud, 1(5), 1-24.

Krug von Nidda, S. (2019). Welchen Einfluss haben betriebliche Selektionskriterien auf den Bewerbungserfolg von Jugendlichen mit Migrationshintergrund? Berufsbildung in Wissenschaft und Praxis, 48(1), 25-29.

Landesamt für Statistik Niedersachsen. (2014). Menschen mit Migrationshintergrund in Niedersachsen. Ergebnisse des Zensus 2011 auf regionaler Ebene. Hannover.

Landesamt für Statistik Niedersachsen. (2019). Regionale Strukturdaten der Betriebe. Auswertungen aus dem Unternehmensregister, Hannover.

McPherson, M., Smith-Lovin, L., \& Cook, J.M. (2001). Birds of a feather: Homophily in social networks. Ann Rev Sociol, 27, 415-444.

MK (Niedersächsisches Kultusministerium). (2019). Hauptschule. Retrieved July 18, 2019, from https://www.mk.niedersachsen.de/startseite/schule/unsere_schulen/allgemein_bildende_schulen/h auptschule/hauptschule-6423.html

Powell, J.J.W., \& Felkendorff, K. (2008). Transitions of people with disabilities beyond secondary education in Austria, Germany, and Switzerland. Background report prepared for the Organisation for Economic Co-operation and Development (OECD), Berlin/Zürich.

Protsch, P. (2013). Höhere Anforderungen in der beruflichen Erstausbildung. WSI-Mitteilungen, 66(1), $15-22$.

Protsch, P. (2017). Getting a foot in the door: How organisational characteristics shape recruitment decisions in the dual apprenticeship system. JEduc Work, 30(6), 585-598.

Protsch, P., \& Solga, H. (2016). The social stratification of the German VET system. J Educ Work, 29(6), 637-661.

Protsch, P., \& Solga, H. (2017). Going across Europe for an apprenticeship? A factorial survey experiment on employers' hiring preferences in Germany. J Eur Soc Policy, 27(4), 387-399.

Riehl, C.M. (2006). Aspekte von Mehrsprachigkeit: Formen, Vorteile, Bedeutung. In M. Becker-Mrotzek et al. (Eds.), Kölner Beiträge zur Sprachdidaktik (pp. 15-23). Duisburg, Gilles \& Francke.

Roth, L.M. (2004). The social psychology of tokenism: Status and homophily processes on Wall Street. Sociol Perspect, 47(2), 189-214.

Scherr, A. (Ed.). (2015). Diskriminierung in der beruflichen Bildung. Beltz Juventa, Weinheim.

Schittenhelm, K. (2005). Primäre und sekundäre Effekte kultureller Praktiken. Der Ausbildungseinstieg junger Migrantinnen im interkulturellen Vergleich. Kölner Zeitschrift für Socziologie und Sozialpsychologie, 57(4), 691-715.

Schneider, J., Yemane, R., \& Wienmann, M. (2015). Diskriminierung am Ausbildungsmarkt. Ausmaß, Ursachen und Handlungsperspektiven. Sachverständigenrat deutscher Stiftungen für Integration und Migration, Berlin. 
Schreyer, F., Bauer, A., \& Kohn, K-H.P. (2015). Betriebliche Ausbildung von Geduldeten. Für den Arbeitsmarkt ein Gewinn, für die jungen Fluchtmigranten eine Chance. IAB Kurzbericht 1/2015, Nürnberg.

Seibert, H., Hupka-Brunner, S., \& Imdorf, C. (2009). Wie Ausbildungssysteme Chancen verteilen. Berufsbildungschancen und ethnische Herkunft in Deutschland und der Schweiz unter Berücksichtigung des regionalen Verhältnisses von betrieblichen und schulischen Ausbildungen. Kölner Zeitschrift für Soziol und Sozialpsychol, 61(4), 595-620.

Söhn, J., \& Marquardsen, K. (2017). Erfolgsfaktoren für die Integration von Flüchtlingen. Forschungsbericht 484. Bundesministerium für Arbeit und Soziales, Berlin.

Solga, H., \& Kohlrausch, B. (2013). How low-achieving German Youth beat the odds and gain access to vocational training-insights from within-group variation. Eur Sociol Rev, 29(5), 1068-1082.

Thijssen, L., Lancee, B., Veit, S., \& Yemane, R. (2019). Discrimination against Turkish minorities in Germany and the Netherlands: Field experimental evidence on the effect of diagnostic information on labour market outcomes. J Ethn Migr Stud. https://doi.org/10.1080/1369183x.2019.1622793

Zschirnt, E. (2019). Evidence of hiring discrimination against the second generation: Results from a correspondence test in the Swiss labour market. J Int Migr Integr. https://doi.org/10.1007/s12134019-00664-1

\section{TRANSLATED VERSION: SPANISH}

Below is a rough translation of the insights presented above. This was done to give a general understanding of the ideas presented in the paper. Please excuse any grammatical mistakes and do not hold the original authors responsible for these mistakes.

\section{VERSION TRADUCIDA: ESPAÑOL}

A continuación se muestra una traducción aproximada de las ideas presentadas anteriormente. Esto se hizo para dar una comprensión general de las ideas presentadas en el documento. Por favor, disculpe cualquier error gramatical y no responsabilite a los autores originales de estos errores.

\section{INTRODUCCIÓN}

Alemania es un excelente ejemplo de una economía coordinada con un gran segmento de la formación profesional (EFP) dentro del sistema educativo nacional. Las empresas son un actor importante en la organización de los aprendizajes en el sistema de FP dual, que tienen lugar tanto en el trabajo en una empresa como en las escuelas ocupacionales. Los jóvenes que buscan esta cualificación tienen que aplicar individualmente a las empresas que ofrecen aprendizajes. Es la empresa, no las escuelas vocacionales, la que puede elegir entre los solicitantes. Por lo tanto, las empresas actúan como guardianes de dicha formación profesional. Este artículo se centra en cómo funciona dicho control de puertas. La correspondencia entre la empresa y el aprendiz está sujeta a mecanismos de mercado, como los procedimientos de contratación de empleados. Esta lógica de selección se caracteriza por la relación entre la oferta y la demanda, por las colas de solicitantes con posiciones más o menos favorables, y por el riesgo de discriminación basada en características ascriptivas como el género o el origen étnico.

En términos legales, no hay condiciones previas con respecto a las credenciales educativas, ya sea el tipo de diploma de escuela secundaria o la calificación promedio alcanzada, con respecto a la mayoría de los tipos de aprendizaje en Alemania. En realidad, sin embargo, esta importante transición en el curso de la vida de los adolescentes de la escuela secundaria a la formación ocupacional depende en gran medida del tipo de diploma con el que se gradúen los solicitantes (véase, por ejemplo, Autorengruppe Bildungsberichterstattung 2018; Baas y Philipps 2019; Protsch y Solga 2016; Erdmann 2016). Dentro del sistema de educación secundaria altamente estratificado en Alemania, los graduados con un diploma de 
escuela secundaria de bajo nivel (Hauptschulabschluss) enfrentan posibilidades muy bajas de comenzar un aprendizaje que aquellos con un diploma de escuela de nivel medio (Realschulabschluss / Mittlere Reife) o un diploma de escuela de alto nivel (Abitur), que también permite el acceso a la educación terciaria. La calificación posteslacial, incluido su nivel y especialización ocupacional, influye fuertemente en las oportunidades de empleo, la calidad del trabajo y la gratificación financiera en su curso de vida posterior.

El tipo de diploma de escuela secundaria que los solicitantes de FP dual pueden presentar a los posibles empleadores sirve a este último como una señal para el capital humano y la capacidad de aprendizaje del solicitante, en cierta medida una señal justificada. El trato desigual basado en características ascriptivas, por el contrario, no es ni legítimo ni legal. En este sentido, los adolescentes de familias inmigrantes o, dependiendo de la operacionalización empírica, de ciudadanía no nativa están en desventaja para acceder a la EFP en Alemania (Autorengruppe Bildungsberichterstattung 2018; Beicht y Walden 2018, 2019; Hunkler 2016; BMBF 2019; ver también varios capítulos en el volumen editado por Scherr 2015). Se han encontrado disparidades étnicas similares en otros países con un fuerte sistema de FP dual como Suiza (Seibert et al. 2009). Además de las altas correlaciones bivariadas, estos estudios muestran que los solicitantes de minorías étnicas enfrentan un mayor riesgo de permanecer sin FP y tener que recurrir a programas preparatorios prevocacionales incluso cuando el rendimiento académico previo está controlado estadísticamente, es decir, cuando los estudios tienen en cuenta el hecho de que los estudiantes de minorías étnicas ya tienen menos oportunidades de alcanzar diplomas de nivel superior y mejores calificaciones que sus pares de ascendencia nativa. Entre los jóvenes con antecedentes migratorios que ya no asisten a la escuela, el $40 \%$ de los jóvenes de 15 a 19 años y el $28 \%$ de los de 20 a 24 años tienen como máximo un diploma de escuela secundaria de bajo nivel. Estas son acciones sustancialmente mayores que entre sus pares nativos (24 y 15\%, respectivamente; ver Autorengruppe Bildungsberichterstattung: Tab. B5-5web, cálculo del autor).

Este artículo se centra en los solicitantes de FP dual que están multiplicados en desventaja en términos de escuelas secundarias de bajo nivel y condición de minoría étnica, e investiga empíricamente el vínculo empresa-solicitante con respecto a las prácticas y los resultados de reclutamiento. Como se explicará en la sección "Perspectivas previas sobre las desventajas étnicas y relacionadas con la educación en la EFP", la investigación ha investigado hasta ahora las disparidades étnicas en la EFP por parte de los solicitantes o de las empresas. Este estudio utiliza una base de datos única que fusiona una encuesta sobre empresas con una sobre solicitantes que se graduaron de escuelas de bajo nivel en el estado regional alemán de Baja Sajonia y aplicaron con éxito o sin éxito a las empresas antes mencionadas.

Para detectar la discriminación, varios estudios han utilizado pruebas de correspondencia con solicitudes falsas [con respecto al aprendizaje en Alemania, ver Protsch (2017) con respecto a las características organizativas y Protsch y Solga (2017) con respecto a la discriminación basada en el estatus de inmigrante; con respecto a las pasantías de estudiantes universitarios en Alemania, ver Kaas y Manger (2012); sobre la discriminación étnica en el mercado laboral, ver Thijssen et al. (2019) para Alemania en comparación con los Países Bajos y Zschirnt (2019) para Suiza]. En comparación con tales experimentos de campo, los datos utilizados aquí reflejan una mayor medida de validez externa porque capturan la acción real de las empresas en términos de reclutamiento real. Por lo tanto, este estudio puede responder mejor a las siguientes preguntas de investigación: ¿en qué medida las preferencias de reclutamiento de las empresas influyen en qué tipos de graduados de escuelas secundarias de bajo nivel seleccionan para el aprendizaje y qué graduados permanecen sin formación profesional? ¿Qué preferencias de empresa interactúan con el alto riesgo de que los adolescentes de familias inmigrantes permanezcan sin un aprendizaje?

Dado que las justificaciones abiertamente racistas para no reclutar a las minorías étnicas son ilegales y (para muchos) socialmente inaceptables, las formas indirectas y menos conscientes de trato desigual parecen más probables. Los estudios cualitativos que utilizan entrevistas en profundidad con actores de empresas responsables de la contratación de aprendices muestran cómo algunos de ellos expresan formas ocultas de prejuicio étnico. Por ejemplo, se refieren a malas experiencias con aprendices "extranjeros" a pesar de que resulta más adelante en la entrevista que nunca han empleado a uno, sino que simplemente han "escuchado" sobre problemas (Imdorf 2007; ver también Schneider et al. 2015: 418 con respecto a los solicitantes de origen turco a empresas alemanas). Como argumentará la sección "Enfoques conceptuales 
de las empresas como guardianes en la FP dual", la preferencia de las empresas por la familiaridad y la homogeneidad dentro de su fuerza laboral, así como las buenas habilidades del idioma alemán, tanto un requisito funcional como un marcador etnonacional, pueden actuar como vías indirectas que conducen a la sobrerrepresentación de aprendices de familias nativas. La sección "Métodos" presenta la base de datos y la operacionalización. La sección "Resultados y discusión" informa la medida en que las empresas comparten puntos de vista estereotipados sobre los migrantes y luego prueba empíricamente dos hipótesis. La sección "Conclusiones" resume los principales hallazgos y ofrece una perspectiva sobre futuras investigaciones y opciones de políticas.

\section{CONCLUSIÓN}

Este artículo ha utilizado un conjunto único de datos interrelacionados entre la empresa y el solicitante para investigar los mecanismos cruciales de discriminación étnica indirecta en la FP dual en Alemania. El análisis arrojó evidencia de cómo funciona el sesgo étnico en el comportamiento de reclutamiento a través de la conexión entre las preferencias de reclutamiento de las empresas y las características atribuidas de los solicitantes de minorías étnicas. Mientras tanto, los modelos multivariantes mantuvieron constantes señales educativas importantes como la participación previa en programas prevocacionales.

Hallazgos cualitativos previos (Imdorf 2007, 2010) sugieren que las empresas prefieren la armonía y la familiaridad en el mundo doméstico de la empresa. Para evitar crear conflictos o terminar prematuramente un aprendizaje, arruinando así su inversión en los solicitantes "equivocados", se cree que las empresas evitan a los de ascendencia extranjera como una "medida preventiva". Sin embargo, la encuesta cuantitativa de la empresa y los solicitantes encontró solo algunas pruebas de que el énfasis relativo de una empresa en qué tan bien los solicitantes "encajan en el equipo" podría tener una influencia en las posibilidades de los adolescentes de minorías étnicas de ser reclutados. Las investigaciones futuras con un mayor número de casos y mejores posibilidades de diferenciación deben probar si el efecto asumido es particularmente relevante para los jóvenes de minorías étnicas con un alto riesgo de estigmatización étnico-racial, como los identificables por un nombre "no alemán" de origen turco o árabe, un color de piel más oscuro o un pañuelo en la cabeza.

En segundo lugar, las empresas que valoran mucho el criterio de selección de la buena expresión verbal pueden organizar su proceso de reclutamiento de una manera que brinde a los solicitantes de familias inmigrantes la oportunidad de demostrar, por ejemplo, en entrevistas de trabajo, que su alemán es competente, en contra de las suposiciones prejuiciosas sobre los inmigrantes y los jóvenes de segunda generación. En cuanto a las empresas para las que las habilidades lingüísticas son algo menos importantes, parece plausible que los prejuicios con respecto a las habilidades lingüísticas de los graduados de minorías étnicas alimenten un escepticismo más amplio sobre ellas, lo que a su vez se traduce en tasas de formación por debajo de la media. Cuando se les preguntó sobre los problemas con los aprendices "más débiles", solo el 5\% de las empresas alemanas que habían tenido aprendices de minorías étnicas nombraron obstáculos relacionados con el idioma en la encuesta de Enggruber y Rützel (2015: 41). Además, en la encuesta aquí analizada, solo el $2 \%$ de las empresas que habían formado previamente aprendices con antecedentes migratorios no volverían a hacerlo.

En términos de limitaciones metodológicas, los principales inconvenientes de la base de datos utilizada aquí son, para el análisis cuantitativo, el pequeño número de casos, así como la falta de representatividad formal. Por lo tanto, sigue sin estar claro en varios casos si la falta de correlaciones estadísticamente significativas entre los factores potencialmente influyentes y la brecha entre la minoría étnica y la mayoría se debe únicamente al tamaño de la muestra o a una similitud sistemática entre los grupos. Aún así, la interconexión de la encuesta de panel sobre los solicitantes con la encuesta de la empresa es un diseño muy adecuado para abordar preguntas de investigación como las de este artículo. La validez externa es claramente mayor que los estudios experimentales que pueden seguir el éxito de las solicitudes "falsas" solo hasta el punto de una invitación a una entrevista de trabajo, pero no al empleo real de los solicitantes, como podría hacer este estudio. Sin embargo, en términos de lecciones metodológicas para el futuro, parece aconsejable agregar una encuesta de empresa de este tipo a un estudio representativo a gran escala ya 
existente con un enfoque especial en las transiciones de la escuela a la FP y el trabajo, como el Estudio del Panel Educativo Nacional Alemán (Cohorte Inicial de $9^{\circ}$ Grado). 15 Además, la investigación futura puede tratar de medir la calidad de las solicitudes escritas (en términos de, por ejemplo, errores tipográficos y estilo de lenguaje adecuado) y su efecto en las invitaciones a entrevistas de trabajo y en el reclutamiento real, ya que muchas escuelas y programas de apoyo enseñan a los estudiantes cómo escribir "buenas" solicitudes.

El análisis reveló que muchas preferencias de reclutamiento medidas en la encuesta muestran poca variación entre las empresas. Esto los hace estadísticamente inadecuados como predictores, aunque los criterios de selección muy comunes, como la confiabilidad y la motivación del candidato (Krug von Nidda 2019: 27), y especialmente su percepción por parte de la empresa, seguramente juegan un papel importante en el proceso de reclutamiento. Aparte de las características estructurales de la empresa (por ejemplo, la relación entre la oferta y la demanda de aprendizaje y el sector económico), información como la contratación de candidatos por parte de la empresa a través de períodos de prácticas o el criterio de selección de la "impresión general" dada por las solicitudes escritas no se consideraban predictores significativos en los modelos de estimación multivariante. El hecho de que muchos criterios nombrados como importantes para la selección de aprendices no discriminen entre los solicitantes de la mayoría étnica y las minorías se refleja en otra encuesta de la empresa: Enggruber y Rützel (2015: 37) tampoco encuentran diferencias significativas entre las empresas que tuvieron o no aprendices de familias inmigrantes durante los 5 años anteriores, ni con respecto a los criterios de selección ni a las sugerencias para mejorar la calidad y la tasa de éxito de la FP dual en General. Algunos elementos que se pensó que potencialmente ponían a los solicitantes de minorías étnicas en desventaja, como el "instinto" (traducción del autor del cuestionario), no muestran una correlación bivariada de la que hablar. La redacción de los puntos es obviamente importante; debe ser sensible a las normas sociales que desalientan al personal de recursos humanos a expresar opiniones que suenan totalmente no meritocráticas o poco profesionales. Estos problemas metodológicos en las encuestas estandarizadas sugieren que la investigación cualitativa, que puede profundizar, todavía es necesaria en este campo. Sin embargo, como Imdorf (2010: 98) ha sugerido, el siguiente paso, por difícil que sea de realizar, debe ser la observación participante en entrevistas de trabajo o en reuniones cuando el personal de recursos humanos discute a los candidatos entrevistados.

Dado que a casi todas las empresas que ya han formado aprendices de familias inmigrantes no les importaría volver a hacerlo, se debe alentar a aquellos sin esa experiencia a que lo intenten. El envejecimiento demográfico y la expansión de la educación terciaria disminuirán aún más el número de solicitantes de FP. Cuanto más corta sea la fila de solicitantes, más dispuestas podrían estar algunas empresas a "probar" a un aprendiz de minoría étnica por primera vez y, por lo tanto, romper el hielo para otros más adelante. Los resultados presentados aquí sugieren que invitar a tales candidatos y escucharlos ya podría contribuir a difundir posibles reservas por parte de la empresa de que las habilidades orales del idioma alemán podrían ser deficientes.

Además, actores importantes en la arena política como la Confederación Alemana de Sindicatos y la Agencia Federal contra la Discriminación han estado exigiendo durante bastante tiempo que al menos las empresas grandes y medianas adopten procedimientos de solicitud anónimos (FAZ 2019), de lo contrario sigue siendo la excepción en Alemania. Tales prácticas de reclutamiento tienden a mejorar la igualdad de oportunidades para varios grupos de riesgo, incluidas las personas con antecedentes migratorios (para la evaluación de un proyecto piloto de este tipo entre las grandes empresas alemanas, véase Krause et al. 2012: 9-10). La mayoría de los solicitantes de FP de minorías étnicas han pasado por el sistema escolar alemán desde el jardín de infantes en adelante y probablemente se beneficiarían de procedimientos de solicitud anónimos, aunque la carrera educativa extranjera de los inmigrantes (como la de los refugiados recientes) difícilmente puede disfrazarse en un CV, lo que apunta a las limitaciones de dicha herramienta. Por último, los Estados de la región deberían invertir más recursos en las oportunidades educativas de los niños inmigrantes y de los hijos de inmigrantes en la educación primaria y secundaria con el fin de aumentar sus posibilidades de acceder a la FP dual. 


\section{TRANSLATED VERSION: FRENCH}

Below is a rough translation of the insights presented above. This was done to give a general understanding of the ideas presented in the paper. Please excuse any grammatical mistakes and do not hold the original authors responsible for these mistakes.

\section{VERSION TRADUITE: FRANÇAIS}

Voici une traduction approximative des idées présentées ci-dessus. Cela a été fait pour donner une compréhension générale des idées présentées dans le document. Veuillez excuser toutes les erreurs grammaticales et ne pas tenir les auteurs originaux responsables de ces erreurs.

\section{INTRODUCTION}

L'Allemagne est un excellent exemple d'économie coordonnée avec un large segment de la formation professionnelle (EFP) au sein du système éducatif national. Les entreprises sont un acteur majeur en ce qui concerne l'organisation des apprentissages dans le système de FEP duale, qui se déroulent à la fois sur le lieu de travail dans une entreprise et dans les écoles professionnelles. Les jeunes qui aspirent à cette qualification doivent postuler individuellement dans les entreprises qui proposent des apprentissages. C'est l'entreprise - et non les écoles professionnelles - qui peut choisir parmi les candidats. Par conséquent, les entreprises jouent le rôle de gardiens de cette formation professionnelle. Cet article se concentre sur la façon dont un tel contrôle fonctionne. L'appariement de l'entreprise et du stagiaire est soumis à des mécanismes de marché tels que les procédures de recrutement des employés. Cette logique de sélection se caractérise par la relation entre l'offre et la demande, par des files d'attente de candidats avec des positions plus ou moins favorables, et par le risque de discrimination fondée sur des caractéristiques ascriptives comme le sexe ou l'origine ethnique.

En termes juridiques, il n'y a pas de conditions préalables concernant les diplômes d'études - qu'il s'agisse du type de diplôme d'études secondaires ou de la note moyenne obtenue - en ce qui concerne la plupart des types d'apprentissage en Allemagne. En réalité, cependant, cette transition importante dans le parcours de vie des adolescents de l'école secondaire à la formation professionnelle dépend beaucoup du type de diplôme avec lequel les candidats obtiennent leur diplôme (voir, par exemple, Autorengruppe Bildungsberichterstattung 2018; Baas et Philipps 2019; Protsch et Solga 2016; Erdmann, 2016). Dans le système d'enseignement secondaire hautement stratifié en Allemagne, les diplômés ayant un diplôme d'études secondaires de bas niveau (Hauptschulabschluss) ont des chances nettement plus faibles de commencer un apprentissage que ceux ayant un diplôme d'études de niveau intermédiaire (Realschulabschluss / Mittlere Reife) ou un diplôme d'études supérieures (Abitur), ce qui permet également l'accès à l'enseignement supérieur. La qualification post-scolaire, y compris son niveau et sa spécialisation professionnelle, influence fortement les possibilités d'emploi, la qualité de l'emploi et la gratification financière dans leur parcours de vie ultérieur.

Le type de diplôme d'études secondaires que les candidats à l'EFP en alternance peuvent présenter à des employeurs potentiels sert à ces derniers de signal pour le capital humain et la capacité d'apprentissage du candidat - dans une certaine mesure un signal justifié. L'inégalité de traitement fondée sur des caractéristiques ascriptives, en revanche, n'est ni légitime ni légale. À cet égard, les adolescents issus de familles immigrées ou, selon l'opérationnalisation empirique, de citoyenneté non autochtone sont désavantagés dans l'accès à l'EFP en Allemagne (Autorengruppe Bildungsberichterstattung 2018; Beicht et Walden 2018, 2019; Hunkler 2016; BMBF 2019; voir aussi divers chapitres du volume édité par Scherr 2015). Des disparités ethniques similaires ont été constatées dans d'autres pays dotés d'un solide système de formation professionnelle duale comme la Suisse (Seibert et al. 2009). Outre les corrélations bivariées élevées, ces études montrent que les candidats issus de minorités ethniques courent un risque plus élevé de rester sans EFP et d'avoir à recourir à des programmes préprofessionnels préparatoires même lorsque les résultats scolaires antérieurs sont statistiquement contrôlés, c'est-à-dire lorsque les études expliquent le fait 
que les étudiants issus de minorités ethniques ont déjà moins de chances d'obtenir des diplômes de niveau supérieur et de meilleures notes que leurs pairs de filiation autochtone. Parmi les jeunes issus de l'immigration qui ne fréquentent plus l'école, $40 \%$ des jeunes de 15 à 19 ans et $28 \%$ des 20 à 24 ans ont tout au plus un diplôme d'études secondaires de bas niveau. Il s'agit de parts nettement plus importantes que parmi les pairs natifs (24 et 15\%, respectivement; voir Autorengruppe Bildungsberichterstattung: Tab. B5-5web, calcul de l'auteur).

Cet article se concentre sur les candidats à la double EFP qui sont de plus en plus désavantagés en termes d'écoles secondaires de bas niveau et de statut de minorité ethnique, et étudie empiriquement le lien entreprise-candidat en ce qui concerne les pratiques et les résultats de recrutement. Comme nous le verrons plus en avant dans la section "Aperçus antérieurs des désavantages ethniques et liés à l'éducation dans l'EFP », la recherche a jusqu'à présent étudié les disparités ethniques dans l'EFP du côté des candidats ou des entreprises. Cette étude utilise une base de données unique qui fusionne une enquête sur les entreprises avec une enquête sur les candidats diplômés d'écoles de bas niveau dans le Land allemand de Basse-Saxe et ayant postulé avec succès ou sans succès aux entreprises susmentionnées.

Afin de détecter la discrimination, plusieurs études ont utilisé des tests de correspondance avec de fausses applications [concernant l'apprentissage en Allemagne, voir Protsch (2017) en ce qui concerne les caractéristiques organisationnelles et Protsch et Solga (2017) concernant la discrimination fondée sur le statut d'immigrant; en ce qui concerne les stages des étudiants en Allemagne, voir Kaas et Manger (2012); sur la discrimination ethnique sur le marché du travail, voir Thijssen et al. (2019) pour l'Allemagne par rapport aux Pays-Bas et Zschirnt (2019) pour Suisse]. Par rapport à de telles expériences sur le terrain, les données utilisées ici reflètent une plus grande mesure de la validité externe car elles capturent l'action réelle des entreprises en termes de recrutement réel. Ainsi, cette étude peut mieux répondre aux questions de recherche suivantes: dans quelle mesure les préférences de recrutement des entreprises influencent-elles les types de diplômés des écoles secondaires de bas niveau qu'elles choisissent pour l'apprentissage et quels diplômés restent sans formation professionnelle? Quelles préférences de l'entreprise interagissent avec le risque élevé que les adolescents issus de familles immigrantes restent sans apprentissage?

Étant donné que les justifications carrément racistes pour ne pas recruter de minorités ethniques sont illégales et (pour beaucoup) socialement inacceptables, des formes indirectes et moins conscientes d'inégalité de traitement semblent plus probables. Des études qualitatives utilisant des entretiens approfondis avec des acteurs d'entreprises responsables de l'embauche d'apprentis montrent comment certains d'entre eux expriment des formes cachées de préjugés ethniques. Par exemple, ils se réfèrent à de mauvaises expériences avec des apprentis « étrangers » même s'il s'avère plus tard dans l'entretien qu'ils n'en ont jamais réellement employé un mais ont simplement « entendu » parler de problèmes (Imdorf 2007; voir aussi Schneider et al. 2015: 418 en ce qui concerne les candidats d'origine turque dans les entreprises allemandes). Comme le soutiendra la section «Approches conceptuelles des entreprises en tant que gardiens de la double EFP », la préférence des entreprises pour la familiarité et l'homogénéité au sein de leur main-d'œuvre ainsi que de bonnes compétences en allemand - à la fois une exigence fonctionnelle et un marqueur ethno-national - peuvent agir comme des voies indirectes conduisant à la surreprésentation des apprentis issus de familles autochtones. La section " Méthodes » présente la base de données et l'opérationnalisation. La section "Résultats et discussion » indique dans quelle mesure les entreprises partagent des points de vue stéréotypés sur les migrants, puis teste deux hypothèses empiriquement. La section « Conclusions » résume les principales conclusions et donne un aperçu des recherches futures et des options politiques.

\section{CONCLUSION}

Cet article a utilisé un ensemble unique de données interdépendantes entre l'entreprise et les demandeurs afin d'étudier les mécanismes cruciaux de discrimination ethnique indirecte dans l'EFP duale en Allemagne. L'analyse a fourni des preuves de la façon dont le biais ethnique dans le comportement de recrutement fonctionne grâce au lien entre les préférences de recrutement des entreprises et les caractéristiques attribuées aux candidats des minorités ethniques. Pendant ce temps, les modèles multivariés 
maintenaient constants des signaux éducatifs importants tels que la participation antérieure à des programmes préprofessionnels.

Des résultats qualitatifs antérieurs (Imdorf 2007, 2010) suggèrent que les entreprises préfèrent l'harmonie et la familiarité dans le monde domestique de l'entreprise. Afin d'éviter de créer des conflits ou de mettre fin prématurément à un apprentissage, gâchant ainsi leur investissement dans les " mauvais » candidats, les entreprises sont censées éviter celles de filiation étrangère en tant que " mesure préventive ». L'enquête quantitative auprès des entreprises et des candidats n'a toutefois trouvé que certaines preuves que l'accent relatif mis par une entreprise sur la façon dont les candidats " s'intègrent dans l'équipe » pourrait avoir une influence sur les chances des adolescents issus de minorités ethniques d'être recrutés. Les recherches futures avec un plus grand nombre de cas et de meilleures possibilités de différenciation devraient vérifier si l'effet supposé est particulièrement pertinent pour les jeunes issus de minorités ethniques présentant un risque élevé de stigmatisation ethnoraciale, tels que ceux identifiables par un nom «non allemand » d'origine turque ou arabe, une couleur de peau plus foncée ou un foulard.

Deuxièmement, les entreprises qui accordent une grande importance au critère de sélection d'une bonne expression verbale peuvent organiser leur processus de recrutement de manière à donner aux candidats issus de familles immigrées la possibilité de prouver, par exemple lors d'entretiens d'embauche, que leur allemand est compétent, contrairement aux préjugés sur les immigrants et les jeunes de deuxième génération. En ce qui concerne les entreprises pour lesquelles les compétences linguistiques sont un peu moins importantes, il semble plausible que les préjugés concernant les compétences linguistiques des diplômés des minorités ethniques alimentent un scepticisme plus large à leur égard, ce qui se traduit par des taux de formation inférieurs à la moyenne. Interrogés sur les problèmes avec les apprentis «plus faibles », seulement $5 \%$ des entreprises allemandes qui avaient eu des apprentis issus de minorités ethniques ont cité des obstacles liés à la langue dans l'enquête d'Enggruber et Rützel (2015: 41). De plus, dans l'enquête analysée ici, seulement $2 \%$ des entreprises qui avaient déjà formé des apprentis issus de l'immigration ne le feraient plus.

En termes de limites méthodologiques, les principaux inconvénients de la base de données utilisée ici sont le petit nombre de cas pour l'analyse quantitative ainsi que le manque de représentativité formelle. Par conséquent, il n'est pas clair dans un certain nombre de cas si l'absence de corrélations statistiquement significatives entre les facteurs potentiellement influents et l'écart entre minorités ethniques et majorité est uniquement due à la taille de l'échantillon ou à une similitude systématique entre les groupes. Néanmoins, l'interconnexion de l'enquête par panel sur les candidats avec l'enquête auprès des entreprises est une conception très appropriée pour aborder des questions de recherche comme celles de cet article. La validité externe est nettement supérieure à celle des études expérimentales qui ne peuvent suivre le succès de " fausses » candidatures qu'au point d'une invitation à un entretien d'embauche mais pas à l'emploi réel des candidats, comme le pourrait le faire cette étude. Cependant, en termes de leçons méthodologiques pour l'avenir, il semble souhaitable d'ajouter une telle enquête auprès des entreprises à une étude représentative à grande échelle déjà existante, avec un accent particulier sur les transitions de l'école à l'EFP et au travail, telles que l'étude allemande du panel national sur l'éducation (cohorte de départ de 9e année). 15 En outre, des recherches futures pourraient tenter de mesurer la qualité des candidatures écrites (en termes de fautes de frappe et de style de langage adéquat) et son effet sur les invitations aux entretiens d'embauche et sur le recrutement réel, car de nombreuses écoles et programmes de soutien enseignent aux étudiants comment rédiger de « bonnes » candidatures.

L'analyse a révélé que de nombreuses préférences de recrutement mesurées dans l'enquête montrent peu de variance entre les entreprises. Cela les rend statistiquement inadaptés en tant que prédicteurs, même si des critères de sélection très courants tels que la fiabilité et la motivation du candidat (Krug von Nidda 2019: 27) - et en particulier leur perception par l'entreprise - jouent certainement un rôle important dans le processus de recrutement. En dehors des caractéristiques structurelles de l'entreprise (par exemple, le rapport entre l'offre et la demande d'apprentissage et le secteur économique), des informations telles que le recrutement de candidats par l'entreprise par le biais de stages ou le critère de sélection de «l'impression générale » donnée par les candidatures écrites ne tenaient pas autant de prédicteurs significatifs dans les modèles d'estimation multivariés. Le fait que de nombreux critères cités comme importants pour la 
sélection des apprentis ne fassent pas de discrimination entre les candidats issus de la majorité ethnique et des minorités est reflété dans une autre enquête auprès des entreprises: Enggruber et Rützel (2015: 37) ne trouvent pas non plus de différence significative entre les entreprises qui ont eu ou n'ont pas eu d'apprentis issus de familles immigrées au cours des 5 années précédentes, ni en ce qui concerne les critères de sélection ni les suggestions pour améliorer la qualité et le taux de réussite de l'EFP en alternance dans Généralités. Certains éléments dont on pensait qu'ils désavantageaient potentiellement les candidats issus de minorités ethniques - comme le « sentiment instinctif » (traduction de l'auteur du questionnaire) - ne montrent aucune corrélation bivariée à proprement parler. Le libellé des articles est évidemment important; il doit être sensible aux normes sociales qui découragent le personnel des RH d'exprimer des opinions qui semblent carrément non méritocratiques ou non professionnelles. Ces problèmes méthodologiques dans les enquêtes standardisées suggèrent que la recherche qualitative, qui peut aller plus loin, est encore nécessaire dans ce domaine. Pourtant, comme Imdorf (2010: 98) l'a suggéré, la prochaine étape, même si elle est difficile à réaliser, devrait être l'observation des participants lors d'entretiens d'embauche ou de réunions lorsque le personnel des RH discute des candidats interviewés.

Étant donné que presque toutes les entreprises qui ont déjà formé des apprentis issus de familles immigrées n'hésiteraient pas à le faire à nouveau, ceux qui n'ont pas une telle expérience devraient être encouragés à essayer. Le vieillissement démographique et l'expansion de l'enseignement supérieur diminueront encore le nombre de candidats à l'EFP. Plus la liste des candidats est courte, plus certaines entreprises pourraient devenir disposées à « essayer » un apprenti appartenant à une minorité ethnique pour la première fois et donc à briser la glace pour d'autres plus tard. Les résultats présentés ici suggèrent que le fait d'inviter de tels candidats et de les écouter pourrait déjà contribuer à dissier les réserves potentielles de l'entreprise selon lesquelles les compétences orales en allemand pourraient être déficientes.

En outre, des acteurs importants dans l'arène politique comme la confédération allemande des syndicats et l'Agence fédérale de lutte contre la discrimination exigent depuis un certain temps qu'au moins les grandes et moyennes entreprises adoptent des procédures de candidature anonymisées (FAZ 2019) - sinon toujours l'exception en Allemagne. De telles pratiques de recrutement tendent à améliorer l'égalité des chances pour divers groupes à risque, y compris les personnes issus de l'immigration (pour l'évaluation d'un tel projet pilote parmi les grandes entreprises allemandes, voir Krause et al. 2012: 9-10). La plupart des candidats des minorités ethniques à l'EFP sont passés par le système scolaire allemand depuis la maternelle et bénéficieraient probablement de procédures de candidature anonymes, bien que la carrière éducative des immigrants à l'étranger (comme celle des réfugiés récents) puisse difficilement être déguisée en CV, soulignant les limites d'un tel outil. Enfin, les États régionaux devraient investir davantage de ressources dans les possibilités d'éducation des enfants immigrés et des enfants d'immigrants dans l'enseignement primaire et secondaire afin d'augmenter leurs chances d'accéder à l'EFP en alternance.

\section{TRANSLATED VERSION: GERMAN}

Below is a rough translation of the insights presented above. This was done to give a general understanding of the ideas presented in the paper. Please excuse any grammatical mistakes and do not hold the original authors responsible for these mistakes.

\section{ÜBERSETZTE VERSION: DEUTSCH}

Hier ist eine ungefähre Übersetzung der oben vorgestellten Ideen. Dies wurde getan, um ein allgemeines Verständnis der in dem Dokument vorgestellten Ideen zu vermitteln. Bitte entschuldigen Sie alle grammatikalischen Fehler und machen Sie die ursprünglichen Autoren nicht für diese Fehler verantwortlich. 


\section{EINLEITUNG}

Deutschland ist ein Paradebeispiel für eine koordinierte Wirtschaft mit einem großen Teil der beruflichen Bildung innerhalb des nationalen Bildungssystems. Unternehmen sind ein wichtiger Akteur bei der Organisation von Ausbildungsberufen im dualen Berufsbildungssystem, die sowohl am Arbeitsplatz in einem Unternehmen als auch in Berufsschulen stattfinden. Junge Menschen, die diese Qualifikation anstreben, müssen sich individuell bei Ausbildungsbetrieben bewerben. Es ist das Unternehmen - nicht die Berufsschulen -, das unter den Bewerbern wählen kann. Daher fungieren die Unternehmen als Torwächter zu einer solchen Berufsausbildung. Dieser Artikel konzentriert sich darauf, wie ein solches Gatekeeping funktioniert. Die Abstimmung von Unternehmen und Auszubildenden unterliegt Marktmechanismen wie z.B. Mitarbeiterrekrutierungsverfahren. Diese Selektionslogik ist gekennzeichnet durch das Verhältnis von Angebot und Nachfrage, durch Bewerberschlangen mit mehr oder weniger günstigen Positionen und durch das Risiko einer Diskriminierung aufgrund von askriptiven Merkmalen wie Geschlecht oder ethnischer Herkunft.

Rechtlich gesehen gibt es für die meisten Ausbildungsarten in Deutschland keine Voraussetzungen für den Bildungsabschluss - sei es die Art des Sekundarschulabschlusses oder die erreichte Durchschnittsnote. In der Realität hängt dieser wichtige Übergang im Lebensverlauf von Jugendlichen von der Sekundarschule in die Berufsausbildung jedoch stark von der Art des Abschlusses ab (siehe z.B. Autorengruppe Bildungsberichterstattung 2018; Baas und Philipps 2019; Protsch und Solga 2016; Erdmann 2016). Innerhalb des stark geschichteten Sekundarschulsystems in Deutschland haben Absolventen mit einem Hauptschulabschluss deutlich geringere Chancen, eine Ausbildung zu beginnen, als Absolventen mit einem Realschulabschluss (Mittlere Reife) oder einem Abitur, der auch den Zugang zu einer tertiären Bildung ermöglicht. Die nachschulische Qualifikation, einschließlich ihres Niveaus und ihrer beruflichen Spezialisierung, beeinflusst stark die Beschäftigungsmöglichkeiten, die Arbeitsplatzqualität und die finanzielle Befriedigung im späteren Lebensverlauf.

Die Art von Sekundarschulabschluss, den Bewerber für eine duale Ausbildung potenziellen Arbeitgebern vorlegen können, dient letzterem als Signal für das Humankapital und die Lernfähigkeit des Bewerbers - gewissermaßen ein berechtigtes Signal. FEine Ungleichbehandlung aufgrund askriptiver Merkmale ist dagegen weder legitim noch rechtmäßig. In diesem Zusammenhang sind Jugendliche aus Migrantenfamilien oder - je nach empirischer Operationalisierung - mit nicht-einheimischer Staatsangehörigkeit beim Zugang zur Berufsbildung in Deutschland benachteiligt (Autorengruppe Bildungsberichterstattung 2018; Beicht und Walden 2018, 2019; Hunkler 2016; BMBF 2019; siehe auch verschiedene Kapitel im Sammelband von Scherr 2015). Ähnliche ethnische Disparitäten wurden in anderen Ländern mit einem starken dualen Berufsbildungssystem wie der Schweiz festgestellt (Seibert et al. 2009). Neben hohen bivariaten Korrelationen zeigen diese Studien, dass Bewerber aus ethnischen Minderheiten einem höheren Risiko ausgesetzt sind, ohne Berufsbildung zu bleiben und auf vorbereitende Berufsvorbereitungsprogramme zurückgreifen zu müssen, selbst wenn frühere akademische Leistungen statistisch kontrolliert werden, d.h. wenn die Studien der Tatsache Rechnung tragen, dass Studenten ethnischer Minderheiten bereits weniger Chancen haben, höhere Diplome und bessere Noten zu erreichen als Gleichaltrige einheimischer Abstammung. Unter den Jugendlichen mit Migrationshintergrund, die nicht mehr zur Schule gehen, haben $40 \%$ der 15 - bis 19-Jährigen und 28\% der 20- bis 24-Jährigen höchstens einen niedrigen Sekundarschulabschluss. Das sind wesentlich größere Anteile als bei nativen Peers (24 bzw. 15\%; siehe Autorengruppe Bildungsberichterstattung: Tab. B5-5web, Autorenrechnung).

Dieser Artikel konzentriert sich auf Bewerberinnen und Bewerber in der dualen Berufsbildung, die in Bezug auf die Sekundarstufe ii. Auf niedrigem Niveau und den Status ethnischer Minderheiten mehrfach benachteiligt sind, und untersucht empirisch die Verbindung zwischen Unternehmen und Bewerbern im Hinblick auf Rekrutierungspraktiken und -ergebnisse. Wie im Abschnitt "Bisherige Erkenntnisse zu ethnischen und bildungsbedingten Benachteiligungen in der Berufsbildung" erläutert, hat die Forschung bisher ethnische Disparitäten in der Berufsbildung auf Seiten von Bewerbern oder Unternehmen untersucht. Diese Studie verwendet eine einzigartige Datenbank, die eine Umfrage unter Unternehmen mit einer 
umfrage unter Bewerbern zusammenführt, die an niedersächsischen Schulen studiert und sich erfolgreich oder erfolglos bei den oben genannten Unternehmen beworben haben.

Um Diskriminierung aufzudecken, haben mehrere Studien Korrespondenztests mit gefälschten Bewerbungen [bezüglich der Ausbildung in Deutschland, siehe Protsch (2017) in Bezug auf organisatorische Merkmale und Protsch und Solga (2017) bezüglich Diskriminierung aufgrund des Einwanderungsstatus; zu Praktika von Hochschulstudenten in Deutschland, siehe Kaas und Manger (2012); zu ethnischer Diskriminierung auf dem Arbeitsmarkt, siehe Thijssen et al. (2019) für Deutschland im Vergleich zu den Niederlanden und Zschirnt (2019) für Schweiz]. Im Vergleich zu solchen Feldexperimenten spiegeln die hier verwendeten Daten ein höheres Maß an externer Validität wider, da sie die tatsächliche Aktion von Unternehmen in Bezug auf die tatsächliche Rekrutierung erfassen. Damit kann

diese Studie folgende Forschungsfragen besser beantworten: Inwieweit beeinflussen die Rekrutierungspräferenzen der Unternehmen, welche Arten von Absolventen von low-level Secondary Schools sie für die Ausbildung auswählen und welche Absolventen ohne Berufsausbildung bleiben? Welche Betrieblichen Präferenzen interagieren mit dem hohen Risiko, dass Jugendliche aus Zuwandererfamilien ohne Ausbildung bleiben?

Angesichts der Tatsache, dass offen rassistische Rechtfertigungen, ethnische Minderheiten nicht zu rekrutieren, illegal und (für viele) sozial inakzeptabel sind, scheinen indirekte und weniger bewusste Formen der Ungleichbehandlung wahrscheinlicher. Qualitative Studien mit Tiefeninterviews mit Akteuren in Unternehmen, die für die Einstellung von Auszubildenden verantwortlich sind, zeigen, wie einige von ihnen verdeckte Formen ethnischer Vorurteile äußern. Sie verweisen zum Beispiel auf schlechte Erfahrungen mit "ausländischen" Auszubildenden, obwohl sich später im Interview herausstellt, dass sie eigentlich nie einen angestellt haben, sondern lediglich von Ärger "gehört" haben (Imdorf 2007; siehe auch Schneider et al. 2015: 418 zu türkischstämmigen Bewerbern bei deutschen Unternehmen). Wie im Abschnitt "Konzeptionelle Ansätze für Unternehmen als Gatekeeper in der dualen Berufsbildung" argumentiert wird, kann die Präferenz der Unternehmen für Vertrautheit und Homogenität innerhalb ihrer Belegschaft sowie gute Deutschkenntnisse - sowohl eine funktionale Anforderung als auch ein ethnonationaler Marker - als indirekte Wege zur Überrepräsentation von Auszubildenden aus einheimischen Familien wirken. Der Abschnitt "Methoden" stellt die Datenbank und die Operationalisierung dar. Der Abschnitt "Ergebnisse und Diskussion" berichtet, inwieweit Unternehmen stereotype Ansichten über Migranten teilen, und testet dann zwei Hypothesen empirisch. Der Abschnitt "Schlussfolgerungen" fasst die wichtigsten Ergebnisse zusammen und gibt einen Ausblick auf zukünftige Forschungs- und Politikoptionen.

\section{SCHLUSSFOLGERUNG}

Dieser Artikel hat einen einzigartigen Satz miteinander verknüpfter Daten aus Unternehmen und Bewerbern verwendet, um entscheidende Mechanismen der indirekten ethnischen Diskriminierung in der dualen Berufsbildung in Deutschland zu untersuchen. Die Analyse lieferte Beweise dafür, wie ethnische Vorurteile im Rekrutierungsverhalten durch die Verbindung zwischen den Rekrutierungspräferenzen von Unternehmen und den zugeschriebenen Merkmalen von Bewerbern ethnischer Minderheiten funktionieren. In der Zwischenzeit hielten multivariate Modelle wichtige Bildungssignale wie die vorherige Teilnahme an berufsvortätigen Programmen konstant.

Bisherige qualitative Erkenntnisse (Imdorf 2007, 2010) legen nahe, dass Unternehmen Harmonie und Vertrautheit in der heimischen Welt des Unternehmens bevorzugen. Um Konflikte zu vermeiden oder eine Ausbildung vorzeitig zu beenden und damit ihre Investition in die "falschen" Bewerber zu verderben, sollen Unternehmen als "vorbeugende Maßnahme" vor solchen ausländischer Abstammung zurückschrecken. Die quantitative Umfrage zu Firmenbewerbern ergab jedoch nur einige Hinweise darauf, dass die relative Betonung eines Unternehmens darauf, wie gut Bewerber "in das Team passen", einen Einfluss auf die Chancen ethnischer Jugendlicher haben könnte, rekrutiert zu werden. Zukünftige Forschungen mit einer höheren Fallzahl und besseren Differenzierungsmöglichkeiten sollten testen, ob der angenommene Effekt besonders relevant für Jugendliche ethnischer Minderheiten mit einem hohen Risiko ethno-rassischer 
Stigmatisierung ist, wie sie beispielsweise an einem "nicht-deutschen" Namen türkischer oder arabischer Herkunft, dunklerer Hautfarbe oder einem Kopftuch erkennbar sind.

Zweitens können Unternehmen, die das Auswahlkriterium des guten verbalen Ausdrucks sehr schätzen, ihren Rekrutierungsprozess so gestalten, dass Bewerber aus Einwandererfamilien die Möglichkeit haben, z.B. in Vorstellungsgesprächen nachzuweisen, dass ihr Deutsch kompetent ist, entgegen vorurteilen Annahmen über Zuwanderer und Jugendliche der zweiten Generation. Was die Unternehmen betrifft, für die sprachliche Fähigkeiten etwas weniger wichtig sind, so erscheint es plausibel, dass Vorurteile über die sprachlichen Fähigkeiten ethnischer Minderheiten zu einer größeren Skepsis gegenüber ihnen führen, was sich wiederum in unterdurchschnittlichen Ausbildungsquoten niederschlagen. Auf die Frage nach Problemen mit "schwächeren" Auszubildenden nannten in der Befragung von Enggruber und Rützel nur 5 $\%$ der deutschen Betriebe, die Auszubildende ethnischer Minderheiten hatten, sprachbezogene Hindernisse (2015: 41). Darüber hinaus würden in der hier analysierten Umfrage nur 2\% der Unternehmen, die zuvor Auszubildende mit Migrationshintergrund ausgebildet hatten, dies nicht noch einmal tun.

In Bezug auf methodische Einschränkungen sind die Hauptnachteile der hier verwendeten Datenbank die - für die quantitative Analyse - geringe Anzahl von Fällen sowie die fehlende formale Repräsentativität. Daher bleibt in einer Reihe von Fällen unklar, ob das Fehlen statistisch signifikanter Korrelationen zwischen potenziell einflussreichen Faktoren und der Kluft zwischen ethnischer Minderheit und Mehrheit nur auf die Stichprobengröße oder auf eine systematische Ähnlichkeit zwischen Gruppen zurückzuführen ist. Dennoch ist die Verknüpfung der Panelbefragung zu Bewerbern mit der Unternehmensbefragung ein sehr geeignetes Design, um Forschungsfragen wie die dieses Artikels anzugehen. Die externe Validität ist deutlich höher als bei experimentellen Studien, die den Erfolg von "fake" Bewerbungen nur bis zur Einladung zu einem Vorstellungsgespräch verfolgen können, nicht aber auf die tatsächliche Beschäftigung der Bewerber, wie es diese Studie könnte. Im Hinblick auf den methodischen Unterricht für die Zukunft erscheint es jedoch ratsam, eine solche Unternehmensbefragung $\mathrm{zu}$ einer bereits bestehenden repräsentativen Großstudie mit besonderem Fokus auf Übergänge von Schule zu Berufsbildung und Beruf wie dem Nationalen Bildungspanel (Startkohorte 9. Klasse) hinzuzufügen. 15 Darüber hinaus könnte die zukünftige Forschung versuchen, die Qualität schriftlicher Bewerbungen (z. B. in Bezug auf Tippfehler und angemessenen Sprachstil) und ihre Auswirkungen auf Einladungen zu Vorstellungsgesprächen und auf die tatsächliche Rekrutierung zu messen, da viele Schulen und Unterstützungsprogramme den Schülern beibringen, wie man "gute" Bewerbungen schreibt.

Die Analyse ergab, dass viele in der Umfrage gemessene Rekrutierungspräferenzen wenig Unterschiede zwischen den Unternehmen aufweisen. Damit sind sie als Prädiktoren statistisch ungeeignet, obwohl sehr häufige Auswahlkriterien wie die Zuverlässigkeit und Motivation des Kandidaten (Krug von Nidda 2019: 27) - und insbesondere ihre Wahrnehmung durch das Unternehmen - sicherlich eine wichtige Rolle im Recruiting-Prozess spielen. Abgesehen von strukturellen Merkmalen des Unternehmens (z. B. das Verhältnis von Angebot und Nachfrage in der Lehrlingsausbildung und der Wirtschaftszweig) waren Informationen wie die Rekrutierung von Kandidaten durch Praktika durch das Unternehmen oder das Auswahlkriterium des "allgemeinen Eindrucks", der durch schriftliche Bewerbungen vermittelt wird, in multivariaten Schätzmodellen nicht als signifikante Prädiktoren zu finden. Dass viele kriterien, die als wichtig für die Auswahl von Auszubildenden genannt werden, nicht zwischen Bewerbern aus der ethnischen Mehrheit und Minderheiten unterscheiden, spiegelt sich in einer weiteren Unternehmensbefragung wider: Enggruber und Rützel (2015: 37) finden auch keinen signifikanten Unterschied zwischen Betrieben, die in den vorangegangenen 5 Jahren Auszubildende aus Zuwandererfamilien hatten oder nicht - weder im Hinblick auf Auswahlkriterien noch auf Vorschläge zur Verbesserung der Qualität und Erfolgsquote der dualen Berufsbildung in Allgemein. Einige Punkte, von denen angenommen wurde, dass sie Bewerber ethnischer Minderheiten potenziell benachteiligen - wie "Bauchgefühl" (Übersetzung des Autors aus dem Fragebogen) - zeigen keine bivariate Korrelation, von der man sprechen könnte. Der Wortlaut der Punkte ist offensichtlich wichtig; Es muss sensibel für soziale Normen sein, die HR-Mitarbeiter davon abhalten, Meinungen zu äußern, die völlig unverdienstkratisch oder unprofessionell klingen. Diese methodischen Probleme in standardisierten Erhebungen legen nahe, dass qualitative Forschung, die tiefer gehen kann, in diesem Bereich noch erforderlich ist. Doch wie Imdorf 
(2010: 98) vorgeschlagen hat, sollte der nächste Schritt - so schwer er zu realisieren ist - die teilnehmerische Beobachtung in Vorstellungsgesprächen oder in Meetings sein, wenn HR-Mitarbeiter interviewte Kandidaten besprechen.

Da fast alle Unternehmen, die bereits Lehrlinge aus Einwandererfamilien ausgebildet haben, nichts dagegen hätten, es noch einmal zu tun, sollten diejenigen ohne solche Erfahrung ermutigt werden, es zu versuchen. Die demografische Alterung und der Ausbau der tertiären Bildung werden die Zahl der Bewerber für eine Berufsbildung weiter verringern. Je kürzer die Bewerberlinie, desto eher könnten einige Unternehmen bereit sein, zum ersten Mal einen Auszubildenden einer ethnischen Minderheit "auszuprobieren" und damit später das Eis für andere zu brechen. Die hier vorgestellten Ergebnisse deuten darauf hin, dass die Einladung und das Zuhören solcher Kandidaten bereits dazu beitragen könnte, mögliche Vorbehalte seitens der Kanzlei zu streuen, dass mündliche Deutschkenntnisse mangelhaft sein könnten.

Darüber hinaus fordern wichtige Akteure der Politik wie der Deutsche Gewerkschaftsbund und die Antidiskriminierungsstelle des Bundes seit geraumer Zeit, dass zumindest große und mittelständische Unternehmen anonymisierte Antragsverfahren einführen (FAZ 2019) - ansonsten in Deutschland noch die Ausnahme. Solche Rekrutierungspraktiken verbessern tendenziell die Chancengleichheit für verschiedene Risikogruppen, einschließlich Personen mit Migrationshintergrund (zur Evaluierung eines solchen Pilotprojekts bei deutschen Großunternehmen siehe Krause et al. 2012: 9-10). Die meisten Bewerber ethnischer Minderheiten für eine Berufsbildung haben das deutsche Schulsystem vom Kindergarten an durchlaufen und würden wahrscheinlich von anonymisierten Bewerbungsverfahren profitieren, obwohl die ausländische Bildungskarriere von Einwanderern (wie die der jüngsten Flüchtlinge) kaum in einem Lebenslauf getarnt werden kann, was auf die Grenzen eines solchen Tools hinweist. Schließlich sollten die Regionalstaaten mehr Ressourcen in die Bildungschancen von Migrantenkindern und Kindern von Einwanderern in der Primar- und Sekundarbildung investieren, um ihre Chancen auf den Zugang zu einer dualen Berufsbildung zu erhöhen.

\section{TRANSLATED VERSION: PORTUGUESE}

Below is a rough translation of the insights presented above. This was done to give a general understanding of the ideas presented in the paper. Please excuse any grammatical mistakes and do not hold the original authors responsible for these mistakes.

\section{VERSÃO TRADUZIDA: PORTUGUÊS}

Aqui está uma tradução aproximada das ideias acima apresentadas. Isto foi feito para dar uma compreensão geral das ideias apresentadas no documento. Por favor, desculpe todos os erros gramaticais e não responsacule os autores originais responsáveis por estes erros.

\section{INTRODUÇÃO}

A Alemanha é um exemplo primordial de uma economia coordenada com um grande segmento de formação educacional profissional (VET) dentro do sistema nacional de ensino. As empresas são um grande ator em relação à organização de aprendizes no sistema VET duplo, que ocorrem tanto no trabalho em uma empresa quanto em escolas ocupacionais. Os jovens que se esforçam por essa qualificação têm que se candidatar individualmente às empresas que oferecem aprendizes. É a empresa - não as escolas profissionalizantes - que podem escolher entre os candidatos. Assim, as empresas atuam como guardiãs de tal formação profissional. Este artigo se concentra em como essa gatekeeping opera. A correspondência entre empresa e trainee está sujeita a mecanismos de mercado, como procedimentos de recrutamento de funcionários. Essa lógica de seleção caracteriza-se pela relação entre oferta e demanda, por filas de

candidatos com posições mais ou menos favoráveis, e pelo risco de discriminação com base em características acritivas como gênero ou origem étnica. 
Em termos legais, não há pré-condições em relação às credenciais educacionais - seja o tipo de diploma de ensino médio ou a nota média alcançada - em relação à maioria dos tipos de aprendizes na Alemanha. Na realidade, porém, essa importante transição no curso de vida dos adolescentes do ensino médio para a formação ocupacional depende muito do tipo de diploma com o qual os candidatos se formam (ver, por exemplo, Autorengruppe Bildungsberichterstattung 2018; Baas e Philipps 2019; Protsch e Solga 2016; Erdmann 2016). Dentro do sistema de ensino médio altamente estratificado na Alemanha, os graduados com diploma de ensino médio de baixo nível (Hauptschulabschluss) enfrentam severamente menores chances de iniciar um estágio do que aqueles com um diploma escolar de nível médio (Realschulabschluss/Mittlere Reife) ou um diploma de ensino médio (Abitur), que também permite o acesso ao ensino superior. A qualificação pós-escolar, incluindo seu nível e especialização ocupacional, influencia fortemente as oportunidades de emprego, a qualidade do trabalho e a gratificação financeira em seu curso de vida subsequente.

O tipo de diploma de ensino médio que os candidatos ao VET duplo podem apresentar a potenciais empregadores serve este último como um sinal para o capital humano e capacidade de aprendizagem do candidato - em certa medida um sinal justificado. Tratamento desigual baseado em características acritivas, em contraste, não é legítimo nem legal. Nesse sentido, adolescentes de famílias de imigrantes ou — dependendo da operacionalização empírica — da cidadania não-nativa são desfavorecidos ao obter acesso ao VET na Alemanha (Autorengruppe Bildungsberichterstattung 2018; Beicht e Walden 2018, 2019; Hunkler 2016; BMBF 2019; veja também vários capítulos no volume editado por Scherr 2015). Disparidades étnicas semelhantes foram encontradas em outros países com um forte sistema duplo VET como a Suíça (Seibert et al. 2009). Além das altas correlações bivariadas, esses estudos mostram que candidatos de minorias étnicas enfrentam maior risco de permanecer sem VEI e ter que recorrer a programas pré-vocacionais preparatórios mesmo quando o desempenho acadêmico anterior é estatisticamente controlado, ou seja, quando os estudos explicam o fato de que os estudantes de minorias étnicas já têm menos oportunidade de alcançar diplomas de nível superior e melhores notas do que os pares de parentes nativos. Entre os jovens com formação migratória que não frequentam mais a escola, $40 \%$ dos jovens de 15 a 19 anos e $28 \%$ dos jovens de 20 a 24 anos têm no máximo um diploma de ensino médio de baixo nível. Estas são ações substancialmente maiores do que entre os pares nativos (24 e 15\%, respectivamente; ver Autorengruppe Bildungsberichterstattung: Tab. B5-5web, cálculo do autor).

Este artigo se concentra em candidatos a duplo VET que são multiplicados em termos de escolas secundárias de baixo nível e status de minoria étnica, e investiga empiricamente a vinculação empresacandidato em relação às práticas e resultados de recrutamento. Como será elaborado na seção "Insights prévios sobre desvantagens étnicas e relacionadas à educação no VET", a pesquisa investigou até agora disparidades étnicas no VET do lado de candidatos ou empresas. Este estudo utiliza um banco de dados único que mescla uma pesquisa sobre empresas com uma sobre candidatos que se formaram em escolas de baixo nível no estado regional alemão da Baixa Saxônia e se candidataram com sucesso ou sem sucesso às empresas mencionadas.

Para detectar discriminação, vários estudos têm usado testes de correspondência com aplicações falsas [sobre o aprendizado na Alemanha, ver Protsch (2017) em relação às características organizacionais e Protsch e Solga (2017) sobre discriminação baseada no status de imigrante; em relação aos estágios de estudantes universitários na Alemanha, ver Kaas e Manger (2012); sobre discriminação étnica no mercado de trabalho, ver Thijssen et al. (2019) para a Alemanha em comparação com os Países Baixos e Zschirnt (2019) para a Alemanha Suíça]. Em comparação com esses experimentos de campo, os dados aqui utilizados refletem uma maior medida de validade externa, pois capturam a real ação das empresas em termos de recrutamento real. Assim, este estudo pode responder melhor às seguintes questões de pesquisa: até que ponto as preferências de recrutamento das empresas influenciam quais tipos de graduados de escolas secundárias de baixo nível eles selecionam para o aprendizado, e quais graduados permanecem sem formação profissional? Quais preferências da empresa interagem com o alto risco de adolescentes de famílias imigrantes permanecerem sem um aprendizado?

Dado que as justificativas racistas para não recrutar minorias étnicas são ilegais e (para muitos) formas socialmente inaceitáveis, indiretas e menos conscientes de tratamento desigual parecem mais prováveis. 
Estudos qualitativos utilizando entrevistas aprofundadas com atores de empresas responsáveis pela contratação de aprendizes mostram como alguns deles expressam formas ocultas de preconceito étnico. Por exemplo, eles se referem a experiências ruins com aprendizes "estrangeiros" mesmo que mais tarde na entrevista eles nunca tenham realmente empregado um, mas apenas "ouvido" sobre problemas (Imdorf 2007; veja também Schneider et al. 2015: 418 no que diz respeito aos candidatos de origem turca a empresas alemãs). Como as abordagens conceituais para as empresas como guardiões na seção vet dupla argumentarão, a preferência das empresas por familiaridade e homogeneidade dentro de sua força de trabalho, bem como boas habilidades de língua alemã — tanto um requisito funcional quanto um marcador etnonacional — podem atuar como caminhos indiretos que levam à superrepresentação de aprendizes de famílias nativas. A seção "Métodos" apresenta o banco de dados e a operacionalização. A seção "Resultados e discussão" relata até que ponto as empresas compartilham visões estereotipadas sobre os migrantes e, em seguida, testa duas hipóteses empiricamente. A seção "Conclusões" resume os principais achados e dá uma visão sobre futuras opções de pesquisa e política.

\section{CONCLUSÃO}

Este artigo utilizou um conjunto único de dados interligados de candidatos a empresas para investigar mecanismos cruciais de discriminação étnica indireta em duplo VET na Alemanha. A análise resultou em evidências de como o viés étnico no recrutamento funciona através da conexão entre as preferências de recrutamento das empresas e as características atribuídas dos candidatos a minorias étnicas. Enquanto isso, modelos multivariados mantinham sinais educacionais importantes, como a participação prévia em programas pré-vocacionais constantes.

Achados qualitativos anteriores (Imdorf 2007, 2010) sugerem que as empresas preferem harmonia e familiaridade no mundo doméstico da empresa. A fim de evitar criar conflitos ou terminar prematuramente um aprendizado, estragando assim seu investimento nos candidatos "errados", acredita-se que as empresas se afastem das de parentesco estrangeiro como uma "medida preventiva". A pesquisa quantitativa de candidatos a empresa, no entanto, encontrou apenas algumas evidências de que a ênfase relativa de uma empresa no quão bem os candidatos "se encaixam na equipe" pode ter uma influência sobre as chances de adolescentes de minorias étnicas serem recrutados. Pesquisas futuras com maior número de casos e melhores possibilidades de diferenciação devem testar se o efeito presumido é particularmente relevante para jovens de minorias étnicas com alto risco de estigmatização etno-racial, como aqueles identificáveis por um nome "não alemão" de origem turca ou árabe, cor da pele mais escura ou um lenço na cabeça.

Em segundo lugar, as empresas que valorizam muito o critério de seleção de boa expressão verbal podem organizar seu processo de recrutamento de forma a dar aos candidatos de famílias imigrantes a oportunidade de provar, por exemplo, em entrevistas de emprego, que seu alemão é proficiente, contrariando suposições preconceituosas sobre imigrantes e jovens de segunda geração. Quanto às empresas para as quais as habilidades linguísticas são um pouco menos importantes, parece plausível que o preconceito em relação às habilidades linguísticas dos graduados em minorias étnicas se alimenta de um ceticismo maior sobre elas, o que, por sua vez, se traduz em taxas de treinamento abaixo da média. Quando questionados sobre problemas com aprendizes "mais fracos", apenas 5\% das empresas alemãs que tinham aprendizes de minorias étnicas nomearam obstáculos relacionados à língua na pesquisa de Enggruber e Rützel (2015: 41). Além disso, na pesquisa aqui analisada, apenas 2\% das empresas que já haviam treinado aprendizes com formação migratória não fariam isso novamente.

Em termos de limitações metodológicas, as principais desvantagens do banco de dados utilizado aqui são o - para análise quantitativa - pequeno número de casos, bem como a falta de representatividade formal. Portanto, ainda não está claro em vários casos se a falta de correlações estatisticamente significativas entre fatores potencialmente influentes e a diferença de maioria étnica minoritária deve-se apenas ao tamanho da amostra ou a uma semelhança sistemática entre os grupos. Ainda assim, interligar a pesquisa do painel sobre os candidatos à pesquisa da empresa é um projeto muito adequado para lidar com questões de pesquisa como as deste artigo. A validade externa é claramente maior do que estudos experimentais que podem acompanhar o sucesso de aplicações "falsas" apenas a ponto de um convite para 
uma entrevista de emprego, mas não para o emprego real dos candidatos, como este estudo poderia. No entanto, em termos de lições metodológicas para o futuro, parece aconselhável adicionar tal pesquisa da empresa a um estudo representativo em larga escala já existente com foco especial em transições escolapara-VET-e-trabalho, como o Estudo Nacional do Painel Educacional Alemão (Coorte Inicial $9^{\circ}$ Ano). 15 Além disso, pesquisas futuras podem tentar medir a qualidade das aplicações escritas (em termos de, por exemplo, erros de digitação e estilo de linguagem adequado) e seu efeito sobre convites para entrevistas de emprego e sobre o recrutamento real, já que muitas escolas e programas de apoio ensinam os alunos a escrever aplicações "boas".

A análise revelou que muitas preferências de recrutamento medidas na pesquisa mostram pouca variância entre as empresas. Isso os torna estatisticamente inadequados como preditores, embora critérios de seleção muito comuns, como a confiabilidade e motivação do candidato (Krug von Nidda 2019: 27) e especialmente sua percepção pela empresa - certamente desempenham um papel importante no processo de recrutamento. Além das características estruturais da empresa (por exemplo, a relação oferta e demanda de aprendizagem e o setor econômico), informações como a contratação de candidatos por meio de estágios ou o critério de seleção da "impressão geral" dada por aplicações escritas não se sustentam como preditores significativos em modelos de estimativa multivariada. $\mathrm{O}$ fato de que muitos critérios apontados como importantes para a seleção de aprendizes não discriminam entre candidatos de maioria étnica e minorias é espelhado em outra pesquisa da empresa: Enggruber e Rützel (2015: 37) também não encontram diferença significativa entre empresas que tinham ou não aprendizes de famílias imigrantes durante os últimos 5 anos - nem no que diz respeito aos critérios de seleção nem a sugestões para melhorar a qualidade e a taxa de sucesso do DUPLO VET em dupla Geral. Alguns itens que foram pensados para potencialmente colocar candidatos de minorias étnicas em desvantagem - como "intuição" (tradução do autor do questionário) não mostram nenhuma correlação bivariada para falar. A redação dos itens é obviamente importante; deve ser sensível às normas sociais que desencorajam o pessoal de RH a expressar opiniões que soem totalmente não meritocráticas ou não profissionais. Esses problemas metodológicos em pesquisas padronizadas sugerem que a pesquisa qualitativa, que pode ir mais fundo, ainda é necessária nesse campo. No entanto, como sugeriu imdorf (2010: 98), o próximo passo — por mais difícil de perceber — deve ser a observação participante em entrevistas de emprego ou em reuniões quando o pessoal do RH discute candidatos entrevistados.

Dado que quase todas as empresas que já treinaram aprendizes de famílias de imigrantes não se importariam de fazê-lo novamente, aqueles sem essa experiência devem ser encorajados a tentar. $\mathrm{O}$ envelhecimento demográfico e a expansão do ensino superior diminuirão ainda mais o número de candidatos ao VET. Quanto menor a linha de candidatos, mais dispostas algumas empresas podem se tornar para "experimentar" um aprendiz de minoria étnica pela primeira vez e, portanto, quebrar o gelo para outros mais tarde. Os resultados aqui apresentados sugerem que convidar tais candidatos e ouvi-los já pode contribuir para difundindo potenciais reservas por parte da empresa de que as habilidades de língua oral alemã poderiam ser deficientes.

Além disso, atores importantes na arena política, como a confederação sindical alemã e a Agência Federal Antidiscriminação, vêm exigindo há algum tempo que pelo menos as grandes e médias empresas adotem procedimentos de aplicação anonimizados (FAZ 2019) - caso contrário, ainda é a exceção na Alemanha. Tais práticas de recrutamento tendem a melhorar a igualdade de oportunidades para vários grupos de risco, incluindo indivíduos com experiência em migração (para a avaliação de tal projeto piloto entre grandes empresas alemãs, ver Krause et al. 2012: 9-10). A maioria dos candidatos de minorias étnicas para o VET passou pelo sistema escolar alemão a partir do jardim de infância e provavelmente lucraria com procedimentos de aplicação anonimizados, embora a carreira educacional estrangeira dos imigrantes (como a dos refugiados recentes) dificilmente possa ser disfarçada em um CV, apontando para as limitações de tal ferramenta. Finalmente, os Estados regionais devem investir mais recursos nas oportunidades educacionais de crianças imigrantes e filhos de imigrantes no ensino fundamental e médio, a fím de aumentar suas chances de acesso ao DUPLO VET. 